\title{
A CRHR1 antagonist prevents synaptic loss and memory deficits in a trauma-induced delirium-like syndrome
}

\author{
Silvia Cursano ${ }^{1,2}$ - Chiara R. Battaglia ${ }^{1,2}$ - Carolina Urrutia-Ruiz ${ }^{1}$ Stefanie Grabrucker ${ }^{3}$ - Michael Schön ${ }^{1}$.

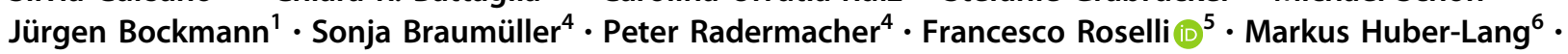 \\ Tobias M. Boeckers $\mathbb{1}^{1}$
}

Received: 27 August 2019 / Revised: 10 January 2020 / Accepted: 20 January 2020 / Published online: 12 February 2020

(c) The Author(s) 2020. This article is published with open access

\begin{abstract}
Older patients with severe physical trauma are at high risk of developing neuropsychiatric syndromes with global impairment of cognition, attention, and consciousness. We employed a thoracic trauma (TxT) mouse model and thoroughly analyzed age-dependent spatial and temporal posttraumatic alterations in the central nervous system. Up to 5 days after trauma, we observed a transient $50 \%$ decrease in the number of excitatory synapses specifically in hippocampal pyramidal neurons accompanied by alterations in attention and motor activity and disruption of contextual memory consolidation. In parallel, hippocampal corticotropin-releasing hormone $(\mathrm{CRH})$ expression was highly upregulated, and brain-derived neurotrophic factor (BDNF) levels were significantly reduced. In vitro experiments revealed that CRH application induced neuronal autophagy with rapid lysosomal degradation of BDNF via the NF- $\mathrm{KB}$ pathway. The subsequent synaptic loss was rescued by BDNF as well as by specific NF-KB and CRH receptor 1 (CRHR1) antagonists. In vivo, the chronic application of a CRHR1 antagonist after TxT resulted in reversal of the observed histological, molecular, and behavioral alterations. The data suggest that neuropsychiatric syndromes (i.e., delirium) after peripheral trauma might be at least in part due to the activation of the hippocampal CRH/NF- $\mathrm{KB} / \mathrm{BDNF}$ pathway, which results in a dramatic loss of synaptic contacts. The successful rescue by stress hormone receptor antagonists should encourage clinical trials focusing on trauma-induced delirium and/or other posttraumatic syndromes.
\end{abstract}

Supplementary information The online version of this article (https:// doi.org/10.1038/s41380-020-0659-y) contains supplementary material, which is available to authorized users.

Tobias M. Boeckers

tobias.boeckers@uni-ulm.de

1 Institute for Anatomy and Cell Biology, Ulm University, AlbertEinstein-Allee 11, 89081 Ulm, Germany

2 International Graduate School in Molecular Medicine, IGradU, 89081 Ulm, Germany

3 Department of Biological Sciences, University of Limerick, Limerick V94 PH61, Ireland

4 Institute for Anesthesiological Pathophysiology, Ulm University, Helmholtzstr. 8/1, 89081 Ulm, Germany

5 Clinic for Neurology, Ulm University, 89081 Ulm, Germany

6 Institute of Clinical and Experimental Trauma-Immunology, Ulm University, 89081 Ulm, Germany

\section{Introduction}

The abrupt decline of mental function (termed delirium) is a common neuropsychiatric syndrome, with a prevalence of $\sim 20 \%$ in the general hospital setting $[1,2]$. In comparison with control patients, patients with delirium have a poorer prognosis and experience an increased length of hospital stay [3], and delirium has been recognized as an important cause of the growing financial burden in an aging society. Delirium is characterized by a general disorientation accompanied by cognitive impairment, changes in arousal and in some cases hallucinations and delusions [4, 5]. The course of the illness is typically short, lasting only a few days to weeks, and delirium is usually reversible [6]. In addition to physical trauma, common causes of delirium include substance intoxication or withdrawal, hyperglycemia, hyperthermia, infections, and brain lesions [7, 8]. Interestingly, advanced age is one of the most predictive risk factors for delirium [9], and following physical trauma, 
a higher multiple organ failure score favors the occurrence of postinjury delirium [7].

Because of the etiological complexity of delirium, the unique disease course in different patients and the problem of defining and operationalizing the syndrome, treatment options, and interventions are limited [10]. Despite its clinical relevance, basic research on trauma-related neuropsychiatric syndromes is complex, and mouse models must be carefully evaluated according to the different types of validity [11]. Therefore, our understanding of the pathophysiology that induces neuropsychiatric alterations after trauma is sparse, and our knowledge on periphery-brain interactions and communication after traumatic events is limited. On the other hand, there have been important studies on the effect of physical stress on the central nervous system (CNS) and the role of stress hormones (especially corticotropin-releasing hormone, $\mathrm{CRH}$ ) in mediating morphological alterations of hippocampal spines and synapses via CRH receptor 1 (CRHR1) activation [12-14]. The authors introduce a $\mathrm{CRH} / \mathrm{calpain} / \mathrm{RhoA}$-dependent mechanism that mediates actin polymerization and depolymerization at synaptic sites. Other lines of evidence support the importance of neurotrophic factors (especially brain-derived neurotrophic factor, BDNF) $[15,16]$ in modulating synaptic and spine plasticity after peripheral stress [17] and/or alterations in stress hormones [18]. Recently, the hypothesis that delirium and hospital-acquired weakness are caused by synaptic dysfunctions was proposed [19].

To elucidate some of the issues discussed above, we employed a peripheral trauma model and searched for morphological and molecular neurobiological correlates/ pathways of posttraumatic disturbances in the brain. To that end, we made use of a blunt chest trauma (TxT) mouse model that presents complex injury of the lung in combination with partial damage to connective and supporting tissue [20]. We screened for structural and molecular alterations in the CNS, especially at the synaptic level, and compared the impact of trauma on young and aged mice at different posttraumatic time intervals.

In this study, we found that $\sim 50 \%$ of pyramidal neuron excitatory synapses within the hippocampal formation were lost, attention and motor activity were altered, and contextual memory in a fear conditioning paradigm was greatly reduced 5 days after trauma in aged animals. Interestingly, the amount of CRH released from hippocampal interneurons was increased, while mature BDNF levels were almost undetectable. Next, we identified and characterized the neuronal CRH-CRHR1-NF- $\mathrm{KB}$ pathway, which induces autophagy and rapid lysosomal degradation of BDNF, to explain local transitional synaptic loss after peripheral trauma. Finally, we rescued TxT-induced synaptic loss and behavioral deficits by chronic treatment with a CRHR1 antagonist. Since we used compounds that are already in medical use, our findings should initiate clinical studies that concentrate on central modulators of stress for the treatment of delirium and other trauma-related insults.

\section{Materials and methods}

\section{Animals}

Male C57BL/6JRj mice (8-10 weeks old (body weight $25 \pm 1.5 \mathrm{~g}$ ) and $22-24$ months old (body weight $30 \pm 1.5 \mathrm{~g}$ )) were group-housed on a 12/12-h light/dark schedule (lights on at 7:0 A.M.) with ad libitum access to food and water before and after blast exposure. All animal experiments were performed in compliance with the guidelines for the welfare of experimental animals issued by the Federal Government of Germany and approved by the Regierungspraesidium Tuebingen and the local ethics committee at Ulm University; ID number: 1233.

\section{Thoracic trauma}

Thoracic trauma (TxT) was always induced during the morning. Mice were anesthetized with a mixture $2.5 \%$ sevoflurane (Sevorane ${ }^{\mathrm{TM}}$, Abbott, Wiesbaden, Germany) and $97.5 \%$ oxygen at a continuous flow of $0.51 \mathrm{~min}^{-1}$ and a FiO2 of 1.0. The mice were fixed to an acrylic glass plate in the supine position, and the abdomen and chest were shaved. Before termination of anesthesia, buprenorphine ( $0.03 \mathrm{mg} \mathrm{kg}^{-1}$ body weight) was injected subcutaneously to provide suitable analgesia. TxT was induced by a single blast wave centered on the thorax as previously described by Ehrnthaller et al. [20]. One group was used as sham controls, and these animals were subjected to the same experimental conditions, but no blast wave was delivered. Analysis was performed $6 \mathrm{~h}$ (for a few experiments), 5 days, 10 days, and 18 days post injury (dpi); at these time points, we collected the brains and blood to perform subsequent analyses. To evaluate the general conditions and estimate the degree of distress, mice were observed every $2 \mathrm{~h}$ after trauma in the operating room, twice a day for the following week and once a day until the end of the experiment.

\section{Golgi staining procedures}

Mice were sacrificed with an overdose of sevoflurane. The brains were removed and prepared for Golgi-Cox staining (FD Rapid GolgiStain ${ }^{\mathrm{TM}}$ Kit). They were then cut sagittally into $150-\mu \mathrm{m}$-thick sections using a vibratome (Microm HM 650) and mounted on gelatin-coated slides. 


\section{Imaging and quantification of dendritic spines}

For the analysis, 9-10 neurons (pyramidal neurons and interneurons) from the CA1 and CA3 hippocampal subregions were analyzed per experimental condition. The apical dendrites in the stratum radiatum/lacunosum were analyzed. Cell types were distinguished based on morphological differences; spines from pyramidal neurons with cell bodies in the pyramidal layer and interneurons with cell bodies in the stratum radiatum were analyzed. Golgi-stained dendritic branches and spines were documented using a BZ-9000 Fluorescence Microscope (KEYENCE Corp.). Image Z-stacks (0.4- $\mu$ m focal steps over the entire thickness of each dendrite) were collected with a $\times 100 / 1.4$ oil lens and reconstructed using ImageJ software. A total of 5-8 dendrites per animal and three animals per group were evaluated. Spine density was expressed as the number of spines per $10 \mu \mathrm{m}$ of dendrite length.

\section{Quantitative real-time PCR}

Isolation of total RNA from mouse brain regions or rat hippocampal cell culture was performed using the RNeasy Mini kit (Qiagen) as described by the manufacturer. Thermal cycling and fluorescence detection were performed using the Rotor-Gene-Q real-time PCR machine (model 2- Plex HRM) (Qiagen). Cycle threshold values were calculated by Rotor-Gene Q software (version 2.0.2). All qRT-PCR reactions were run in triplicate for each time point and condition. Real-time quantitative PCR was carried out using oligonucleotides to investigate the expression of CRH, CRHR1, CRHR2, BDNF, and TrkB (validated primer pairs, Quantitect Primer Assay, Qiagen).

\section{Primary antibodies}

The primary antibodies used for immunocytochemistry are described below. Shank2 (1:500) ("ppI-SAM pabSA5192") was previously characterized [21-23]. The following primary antibodies were purchased from commercial suppliers: Vglut1 (1:500, Synaptic Systems GmbH, \#135304), Gephyrin (1:500, Synaptic Systems GmbH, \#147003), Vgat (1:500, Synaptic Systems GmbH, \#131011), Iba1 (1:250, Wako Chemical GmbH, \#NCNP24), GFAP (1:500, SigmaAldrich, \#G3893), NeuN (1:1000, Millipore, \#MAB377), C1q (1:1000, Abcam, \#182451), CRH (1:1000, Abcam, \#8901), p62 (1:500, Abcam, \#56416), Map2 (1:500, EnCor Biotechnology Inc., \#CPCA-MAP2), Synaptotagmin-1 (1:500, Synaptic Systems, \#105311C5), ATG5 (Abcam, \#AB108327), NF-кB p65 (1:500, Santa Cruz, \#SC8008), phospho-NF-кB (Thr435) (1:500, Thermo Fisher Scientific, \#PA5-37724), phospho-IкB $\alpha$ (Ser32) (1:500, Cell Signaling, \#2859), CRHR1 (1:500, Everest Biotech, \#EB08035), and ChAT (1:250, Synaptic System, \#297015).
The following primary antibodies were used for western blotting: Shank2 (1:2000) ("ppI-SAM pabSA5192"), Synapsin 1/2 (1:1000, Synaptic Systems GmbH, \#160003), Gephyrin (1:500, Synaptic Systems GmbH, \#147003), Vgat (1:500, Synaptic Systems GmbH, \#131011), BDNF (1:500, Abcam, \#203573), C3b/iC3b/C3c (1:200, Hycultec GmbH, \#HM1065), Calpain-1 (1:500, Abcam, \#39170), Actin (1:250000, Sigma-Aldrich, \#A2228), Beclin-1 (BCN-1) (1:500, Novus Biologicals, \#NB500249), LC3 (1:1000, Cell Signaling, \#4599), and Lamp-2 (1:500, Thermo Fisher Scientific, \#PA1655).

\section{Secondary antibodies}

The secondary antibodies used for immunocytochemistry were coupled to Alexa Fluor ${ }^{\circledR} 488$, 568, or 647 (1:500, all from Life Technologies). The secondary antibodies used for western blotting were HRP-conjugated (1:1000 Dako, Glostrup, Denmark).

\section{Slice preparation and immunohistochemistry}

Animals were anesthetized (25\% ketamine and 5\% xylazine solubilized in a $\mathrm{NaCl}$ solution) and perfused with $25 \mathrm{ml}$ cooled phosphate buffer saline (PBS) and $50 \mathrm{ml} 4 \%$ PFA. Then, the brains were treated as previously described by Heise et al. [24]. Images of immunostained sections were taken using an upright fluorescence microscope (Axioskop, Zeiss) and Axiovision software (Zeiss). For in vivo studies, a confocal microscope (Leica SP5 or Leica SPE confocal microscope with a $\times 40$ or $\times 63$ objective) was used. For the magnified images, Fiji ImageJ (National Institute of Health, USA) and Bitplane Imaris software were used.

\section{Immunocytochemical analysis}

Qualitative immunocytochemical analysis was carried out on three male mice. At least three coronal slices per animal were stained with each antibody/antibody combination to assure the representativeness of the staining. The intensity of immunostaining and colocalization were then analyzed using Fiji ImageJ and Bitplane Imaris software.

\section{Western blotting}

Mice were sacrificed with an overdose of sevoflurane. Subcellular fractionation of brain tissue was performed as previously described [25].

\section{Transmission electron microscopy}

Mice were perfused with $20 \mathrm{ml}$ of solution 1 ( $0.5 \%$ heparinized saline solution) followed by $50 \mathrm{ml}$ of solution $2(2 \%$ 
paraformaldehyde, $2.5 \%$ glutaraldehyde, and $1 \%$ saccharose in 0.1 cacodylate phosphate buffer, $\mathrm{pH}$ 7.4). After perfusion, the mouse brains were dissected and postfixed in solution 3 (2\% glutaraldehyde and $1 \%$ saccharose in 0.1 cacodylate buffered saline) at $4{ }^{\circ} \mathrm{C}$ overnight. The regions of interest (CA1 and CA3) were dissected in $1-\mathrm{mm}^{2}$ pieces using a stereomicroscope. Then, the specimens were washed in $0.1 \mathrm{M}$ PBS, postfixed with $2 \%$ osmium tetroxide for $1 \mathrm{~h}$ and dehydrated in an ascending propanol series $(30 \%, 50 \%, 70 \%$, and $90 \%)$. In addition, uranyl acetate diluted in denatured ethanol was used to contrast the specimens for $30 \mathrm{~min}$ at $37^{\circ} \mathrm{C}$. The epon-embedded specimens were cut into $0.5-\mu \mathrm{m}$ sections with an ultratome, and the semithin sections were stained with toluidine blue. The defined subregions were cropped from the epon-embedded pieces, cut into ultrathin sections $(70-80 \mathrm{~nm})$ and collected on 300 mesh copper grids.

For the hippocampal neurons, transmission electron microscopy (TEM) was performed as previously reported [26].

\section{Transmission electron microscopy analysis}

All analyzed specimens were investigated on a Jeol JEM 1400 transmission electron microscope at $120 \mathrm{kV}$. A magnification of $\times 25,000$ was chosen to study synapses and the density of postsynaptic densities (PSDs) within an image section. Generally, synapses were counted within an area of $35.8 \mu \mathrm{m}^{2}$. A magnification of $\times 80,000$ was further applied to measure the PSD length and thickness. Only artifact-free synapses with clearly identifiable PSDs and presynaptic and postsynaptic terminals were selected for analysis. ImageJ software was used to determine the length, thickness, and volume of the PSDs. For the analysis of the SV density, membrane-associated vesicles and presynaptic terminal size were manually traced in ImageJ. Multivesicular bodies (MVBs) were identified as roundshaped vesicles surrounded by a single membrane that enclosed a variable number of small spherical vesicles within a matrix, according to the published criteria [27].

\section{Culturing and immunolabeling of rat hippocampal/ cortical neurons for conventional fluorescence imaging}

Primary rat embryo hippocampal/cortical cell culture and immunolabeling procedures were previously described by Schoen et al. [28].

\section{Image acquisition and analysis of rat hippocampal neurons}

Images were obtained with an upright fluorescence microscope (Zeiss Axioskop 2 and Zeiss Imager Z1 with an apotome, Zeiss, Germany). Pictures were taken with Axiovison 4.7.1 software (Zeiss, Germany). Three different dendrites from three different neurons from three different wells were analyzed for each condition using Bitplane Imaris software.

\section{Experimental and pharmacological design}

The CRH peptide (Bachem \#H-2435) was maintained at a stock solution of $100 \mu \mathrm{M}$ in sterile water, and then diluted to the desired concentration in NBM plus B27 just before use. Dissociated neurons on glass coverslips were incubated in 6or 24-well plates at $36^{\circ} \mathrm{C}$ for $30 \mathrm{~min}$. The selective CRH receptor blocker NBI30775 (Hycultec GmbH, \#HY-14127) and a selective GR blocker (Sigma-Aldrich, \#RU38486) were dissolved in sterile DMSO, sonicated and used at a final concentration of $100 \mathrm{nM}$. BDNF (Peprotech \#450-02) was prepared to a final concentration of $10 \mu \mathrm{g} / \mathrm{ml}$. The blockers were applied alone for $5 \mathrm{~min}$ to allow the compound to bind the receptors. This was followed by application of a solution containing (1) NBI30775 and CRH or (2) NBI30775, RU38486, and CRH, and BDNF was immediately applied in combination with $\mathrm{CRH}$ for another $30 \mathrm{~min}$.

\section{Phospho-protein array}

Screening of 1318 phospho-proteins (Phospho Explorer Antibody Array \#KAS02) was performed in hippocampal neurons under two experimental conditions: vehicle and $100 \mathrm{nM}$ CRH. The detection of antibody arrays was performed in a fluorescence slide scanner (Genepix 4000B microarray scanner, Molecular Devices). The 16-bit images were analyzed using GenePixPro 6.1 software. For quantification, four replicates for each antibody and values from two independent experiments were used. The values were calculated relative to the average value of the corresponding control. Protein expression changes of $>35 \%$ were considered relevant.

\section{Synaptotagmin assay}

After $30 \mathrm{~min}$ of incubation with CRH with or without antagonist(s)/BDNF, synaptotagmin-1 (1:500) was administered for $30 \mathrm{~min}$. Then, DIV14 neurons from all the experimental groups were fixed for ICC or rapidly processed for western blot analysis.

\section{NF-KB activation blockage}

After 30 min of incubation with CRH, JSH23 (Abcam, \#Ab144824) was added at a final concentration of $10 \mu \mathrm{M}$ for $30 \mathrm{~min}$ to prevent NF- $\mathrm{KB}$ translocation to the nucleus. Then, neurons were fixed for ICC or rapidly processed for western blot analysis. To demonstrate the validity of the 
system, two other NF-kB activation blockers were tested: lactacystin (Abcam, \#Ab141411) and SC-514, an IKKbeta inhibitor (Abcam, \#ab144415).

\section{Lysosomal inhibition and reagents}

DIV14 rat hippocampal neurons were treated with $100 \mathrm{nM}$ $\mathrm{CRH}$ for $30 \mathrm{~min}$ in triplicate. Then, $50 \mu \mathrm{M}$ leupeptin hemisulfate (Biomol, CAS \#103476-89-7) and $5 \mu \mathrm{M}$ E64 (Sigma-Aldrich, \#E3132) were added for $30 \mathrm{~min}$. Then, neurons from all the experimental groups were rapidly processed for western blot analysis.

\section{Behavioral test}

Fear Conditioning System 46103 from Noldus/Ugo Basile was used to perform the trace cued and contextual test. The fear conditioning procedure was conducted over 3 days. During the first day (training day), the animals were placed in a unique context $(25 \times 17 \times 17$ box $)$, and after $2 \mathrm{~min}$ of acclimation, they were exposed to a mild foot-shock $(0.5 \mathrm{~mA}, 2 \mathrm{~s})$ followed $(20 \mathrm{~s}$ later) by a tone $(85 \mathrm{~dB}$, $2700 \mathrm{~Hz}, 20 \mathrm{~s}$ ). The CS-US pairing was repeated five times. During the second day (auditory memory), the animals were placed in a different context, and after 2 min of acclimation, they were exposed three times to a tone with 2-min intervals $(85 \mathrm{~dB}, 2700 \mathrm{~Hz}, 20 \mathrm{~s})$. On the third day (contextual memory), the animals were exposed to the same context as that used for the training day for $8 \mathrm{~min}$. On the fourth day, TxT was induced. Upon exposure to the same context or cue 5 or 18 days after TxT, animals exhibited a variety of fear responses, including freezing behavior [29, 30]. Freezing behavior was assessed as the percentage of time spent freezing (measured $1 \mathrm{~min}$ after the tone using Ethovision 12 software). Freezing was defined as a complete lack of movement, except for respiration [29, 30].

\section{In vivo antagonist administration}

The selective CRH receptor blocker NBI30775 (Hycultec $\mathrm{GmbH}$, \#HY-14127) and a selective GR blocker (SigmaAldrich, \#RU38486) were dissolved into sterile 70\% PEG. NBI30775 $(1 \mathrm{mg} / \mathrm{kg})$ was administered subcutaneously immediately and 2, 6, and $10 \mathrm{~h}$ after TxT trauma and twice a day for 1 week. RU38486 (20 mg/kg) was administered subcutaneously immediately and $12 \mathrm{~h}$ after trauma and twice a day for 1 week. The TCCF test was performed as described above.

\section{Statistical analysis}

The results are presented as the mean $\pm \mathrm{SEM}$. CRH/ synapse-related immunostaining and all immunoblots were normalized to the value of the sham group (equal to 1 ) or, for the in vitro experiments, were normalized to the value of the vehicle group (equal to 1). One-way ANOVA followed by Bonferroni's multiple comparison post hoc test was performed for most of the experiments to determine significant differences between experimental means. Some experiments were analyzed using two-tailed unpaired $T$-test or one-way ANOVA followed by the Dunnett post hoc test. The biological replicates from three mice were used for experiments with 2-3 technical replicates, except for the behavioral experiments, in which 6-8 mice were used. For the in vitro experiments, the biological triplicates were derived from 3-5 technical replicates. The 95\% confidence interval was considered statistically significant. GraphPad Prism 7.0 was used to perform all statistical analyses.

\section{Results}

\section{Thoracic trauma (TxT) leads to a selective loss of hippocampal excitatory synapses but no memory deficits in young mice}

We analyzed the spine density of hippocampal pyramidal neurons and interneurons in the CA1 and CA 3 regions of young mice on days 5, 10, and 18 after TxT (Fig. 1a). In pyramidal neurons, the number of dendritic spines was significantly lower (up to 50\%) at 5 and $10 \mathrm{dpi}$, and there were only moderate changes in spine morphology (Supplementary Fig. 1). A general recovery of spine loss was observed after 18 dpi (Fig. 1b). In contrast, no significant changes in spine density were observed in hippocampal interneurons (Fig. 1b). Co-immunostaining with antibodies directed against the excitatory postsynaptic marker Shank2 and the presynaptic protein Vglut1 revealed that spine reduction was accompanied by a loss of synaptic contacts in the CA1 and CA3 regions at 5 and $10 \mathrm{dpi}$ and that this was recovered at 18 dpi (Fig. 1c). This was observed along the whole dorsal to ventral extent of the hippocampal structure (see Supplementary Materials Section 1.3). The results were confirmed by western blot analysis in homogenates and P2 hippocampal fractions; Shank2- and Synapsin 1/2-levels were unchanged in the homogenates (Fig. 1d) but significantly downregulated in synaptic P2 fractions at 5 and 10 dpi. Consistent with these findings, the analysis of excitatory synapses in the hippocampal CA3 region by TEM demonstrated a significant decrease in the number of excitatory synapses. Interestingly, the gross morphology of the remaining synapses after trauma was unchanged, as revealed by the length, thickness, and volume $(\mathrm{V}=$ (length/ $2)^{2} \times$ thickness $\times \pi$ ) of the PSDs (Fig. 1e). In contrast to these findings, the number of excitatory synapses in the cortex (Fig. 1f) was unchanged, as revealed by the 
A

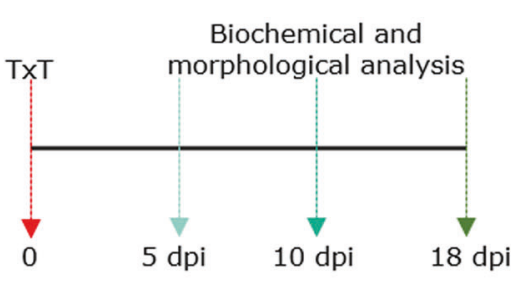

C
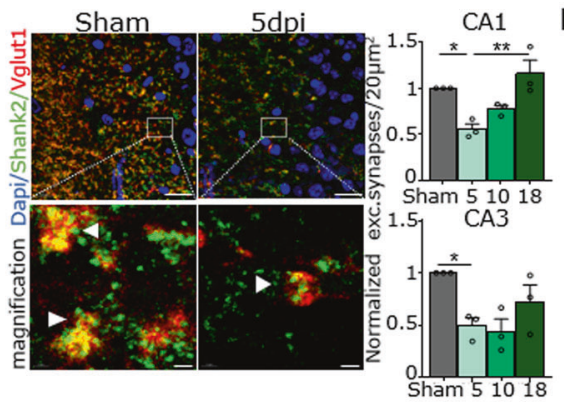

E

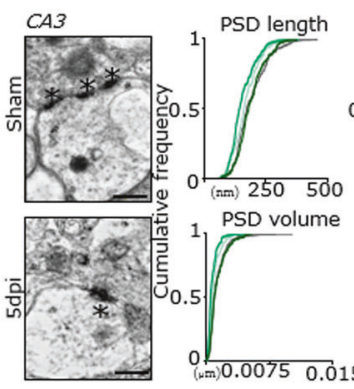

G
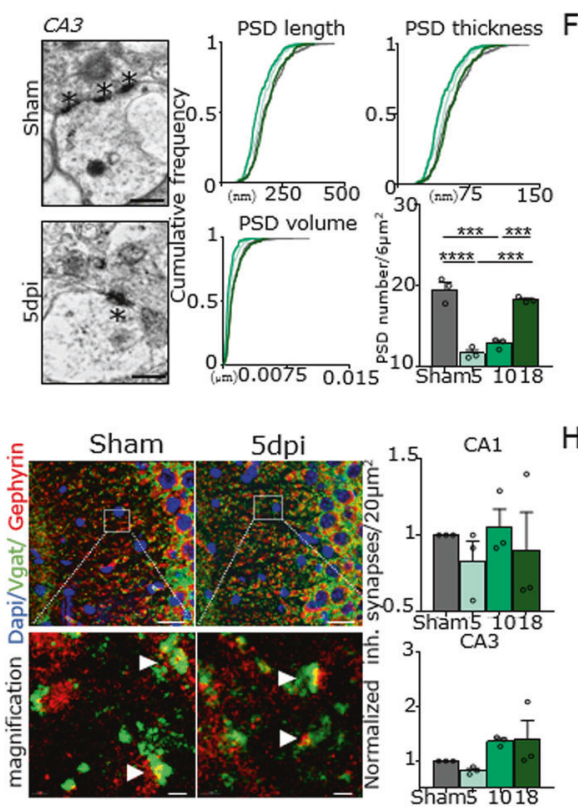

B

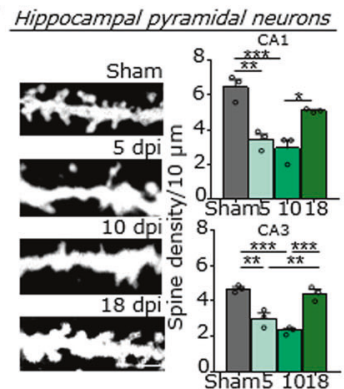

Hippocampal pyramidal neurons

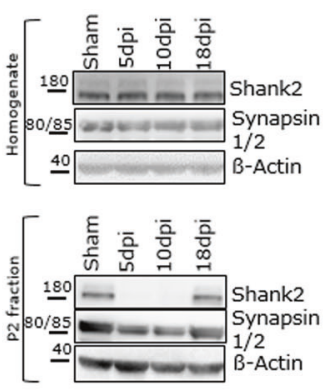

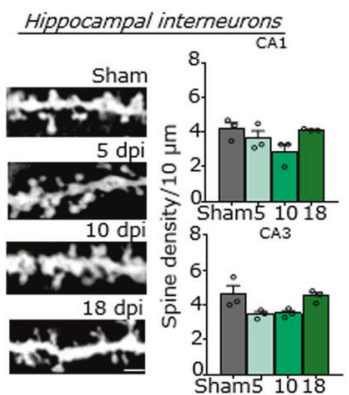
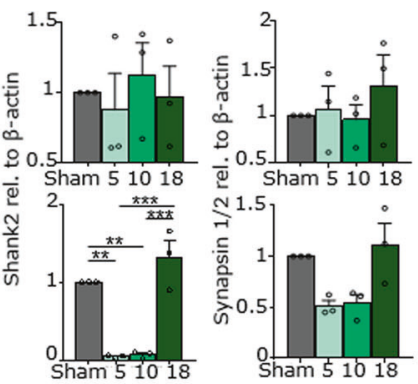
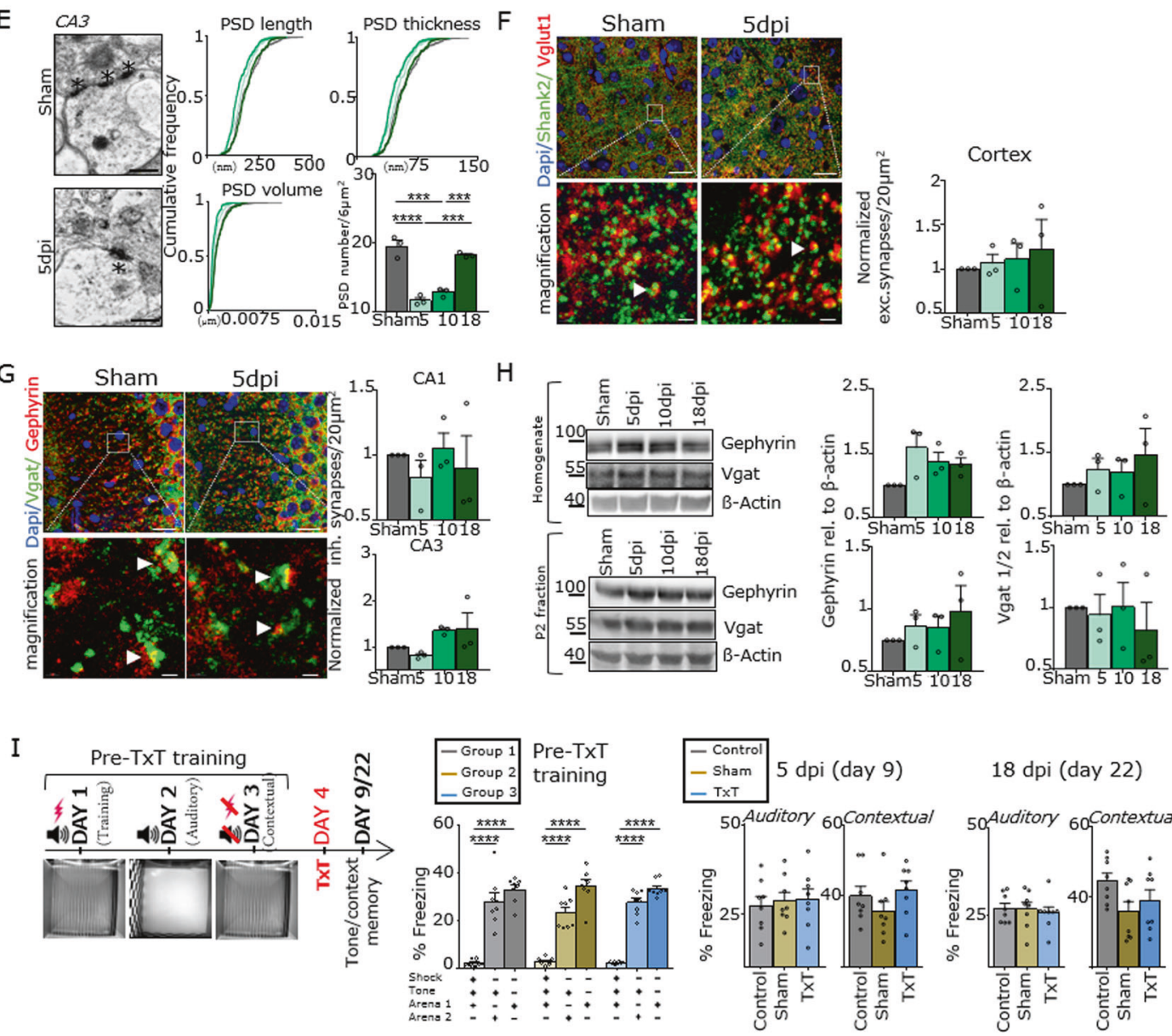

18 dpi (day 22)

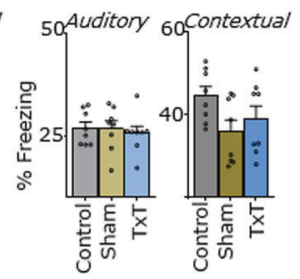

expression of the synaptic marker proteins Shank2 and Vglut1. Moreover, analysis of inhibitory synapses by the pre- and postsynaptic marker proteins Gephyrin and Vgat showed no significant changes in synapse number and/or protein expression in the hippocampal CA1 and CA3 regions (Fig. 1g, h). Finally, young mice were exposed to 
Fig. 1 Loss of hippocampal excitatory synapses after TxT. a Schematic design of the experimental setup. b Analysis of spine density using Golgi staining in (left) pyramidal neurons and (right) interneurons (scale bar $=3 \mu \mathrm{m}$ ). $\mathbf{c}$ Immunohistochemical (IHC) staining and quantification of excitatory synapses in the hippocampus using the postsynaptic marker Shank2 (green) and the presynaptic marker Vglut1 (red) (scale bar $=15 \mu \mathrm{m}$, magnification scale bar $=1 \mu \mathrm{m}$ ). Arrowheads indicate synaptic colocalization. d Western blot analysis and quantification of hippocampal homogenates and the P2 fraction using antibodies directed against the postsynaptic protein Shank2 and the presynaptic marker Synapsin 1/2. e The overall number and ultrastructure of excitatory synapses within the CA3 region. Asterisks indicate the postsynaptic density (PSD) (scale bar $=0.5 \mu \mathrm{m}$ ). f IHC staining and quantification of excitatory synapses in the cortex using Shank2 (green) and Vglut1 (red) (scale bar $=15 \mu \mathrm{m}$, magnification scale bar $=1 \mu \mathrm{m})$. Arrowheads indicate synaptic colocalization of the proteins. $\mathbf{g}$ IHC to detect inhibitory synapses in the hippocampus using the postsynaptic marker Gephyrin (red) and the presynaptic marker Vgat (green). Relative quantification of coimmunostained synaptic puncta (arrowheads) (scale bar $=15 \mu \mathrm{m}$, magnification scale bar $1 \mu \mathrm{m})$. h Western blot analysis and quantification of hippocampal homogenates and P2 fraction for Gephyrin and Vgat. $N=3$. The error bars represent the SEMs; one-way ANOVA with Bonferroni's post hoc comparison test was performed $(* P \leq 0.05$, ** $P \leq 0.005$, ***P $\leq$ $0.0005, * * * * P \leq 0.0001)$. i The trace cued and contextual fear conditioning (TCCF) test protocol. The TCCF test was performed before thoracic trauma, and an auditory and contextual memory test was performed 5 and 18 days after trauma (the results of the auditory memory test and contextual memory test were compared among each group). $N=8$; the error bars represent the SEMs; one-way ANOVA with Bonferroni's post hoc comparison test was performed $(P \leq 0.05$, $* * P \leq 0.005, * * * P \leq 0.0005, * * * * P \leq 0.0001)$.

cue/contextual fear conditioning 5 and 18 days after TxT. No significant differences were observed between the experimental groups with respect to the freezing response (measured $1 \mathrm{~min}$ after the tone) (Fig. 1i).

\section{Screening for potential mechanism(s) involved in synaptic loss reveals a potential role of trauma- related alterations in hippocampal CRH and BDNF expression}

By using a broad screening approach to identify potential mechanisms responsible for hippocampal synaptic loss, we analyzed the potential loss of hippocampal neurons but did not find any difference between the experimental through (Supplementary Fig. 2A, G). Next, we explored the involvement of local inflammation by histological analysis of microglial (Supplementary Fig. 2B, H) and astroglial activation (Supplementary Fig. 2C, I). We observed a slight increase in microglia in the motile stage in the CA1 and CA3 regions after 10 days; however, the number and intensity of GFAP (glial fibrillary acidic protein)-positive astroglia remained unchanged. Next, we investigated the putative involvement of the complement system by using $\mathrm{C} 1 \mathrm{q}$ as a marker of the classical complement cascade and $\mathrm{C} 3 \mathrm{~b}$ as a crucial marker of complement activation.
Interestingly, C1q levels were downregulated at $5 \mathrm{dpi}$, while C3b-positive structures remained unaltered (Supplementary Fig. 2E, K). Moreover, since there is good evidence that cholinergic hypoactivity might be involved in cognitive dysfunction during delirium [31], we investigated the expression of ChAT-positive neurons in the medial septum and hippocampus (CA1 and CA3 regions). No difference in terms of the number of ChAT-positive cholinergic neurons was found between young and aged mice after TxT (Supplementary Fig. 2D, J). In addition, vital parameters after trauma did not show any significant changes, and the expression of the hypoxia-induced factor (Hif-1alpha) was not detected in the brain (data not shown). Finally, we closely analyzed the stress axis in the hippocampus first by analyzing the mRNA expression of CRH and its receptors (Fig. 2a). Here, we found that CRH mRNA was highly upregulated 10 and 18 days after trauma and CRHR1 mRNA was slightly increased at $5 \mathrm{dpi}$, while CRHR2 expression remained unchanged among the different groups. Next, we investigated the expression of CRH by evaluating the intensity of CRH-positive puncta by confocal imaging (Fig. 2a) and found that $\mathrm{CRH}$ expression was significantly enhanced 5 and 10 days after trauma. Moreover, we investigated the corticosterone plasma level in sham and trauma-exposed mice and detected a significant increase in the concentration $(\mathrm{ng} / \mathrm{ml}) 6 \mathrm{~h}$ and $5 \mathrm{dpi}$ (Supplementary Fig. 2F). Since it has been demonstrated that stress influences the expression of different neurotrophic factors [32], we screened for alterations in neurotrophin expression and found by western blot analysis that BDNF was undetectable in the hippocampal region at 5 and $10 \mathrm{dpi}$; on the other hand, there was only a slight decrease in BDNF mRNA after 5 days and a strong increase in TrkB mRNA after 10 days. In contrast, when we analyzed CRH and BDNF expression in the cortex, we did not observe any alteration in mRNA and/or protein expression (Fig. 2b).

\section{Unraveling the direct CRH-NF-KB-BDNF autophagy pathway that results in a rapid loss of excitatory synapses}

\section{CRH induces BDNF downregulation and synaptic loss in vitro}

To study the in vivo findings in more detail, we employed a primary hippocampal/cortical culture system in which the expression of $\mathrm{CRH}$ and its receptors was comparable with that on day 14 after plating (Supplementary Fig. 3A). First, we tested the effect of CRH on the number of excitatory synapses $30 \mathrm{~min}$ after treatment with $\mathrm{CRH}(100 \mathrm{nM})$ and found a significant decrease of $\sim 60 \%$ in the number of synapses (Fig. 3a and Supplementary Fig. 3B). Next, we analyzed whether these changes were also accompanied by 
Fig. 2 Upregulation of hippocampal CRH. a Expression (left) of CRH, CRHR1, and CRHR2 mRNA relative to HMBS; IHC for CRH (green) (scale bar $=15 \mu \mathrm{m}$, magnification scale bar $=2 \mu \mathrm{m})$ and quantification of CRHpositive puncta (right) in the hippocampal formation. Western blot analysis of BDNF (P2 fraction) and the relative quantification of the expression of BDNF and its receptor TrkB relative to HMBS. b CRH, CRHR1, and CRHR2 mRNA expression, IHC for CRH (green, scale bar $=15 \mu \mathrm{m}$, magnification scale bar $=2 \mu \mathrm{m}$ ), immunoblot of BDNF (P2 fraction) and the relative quantification of BDNF and TrkB expression relative to HBMS in cortical tissue. Oneway ANOVA with Bonferroni's post hoc comparison test was performed $(* P \leq 0.05, * * P \leq$ $0.005, * * * P \leq 0.0005, * * * * P \leq$ 0.0001).

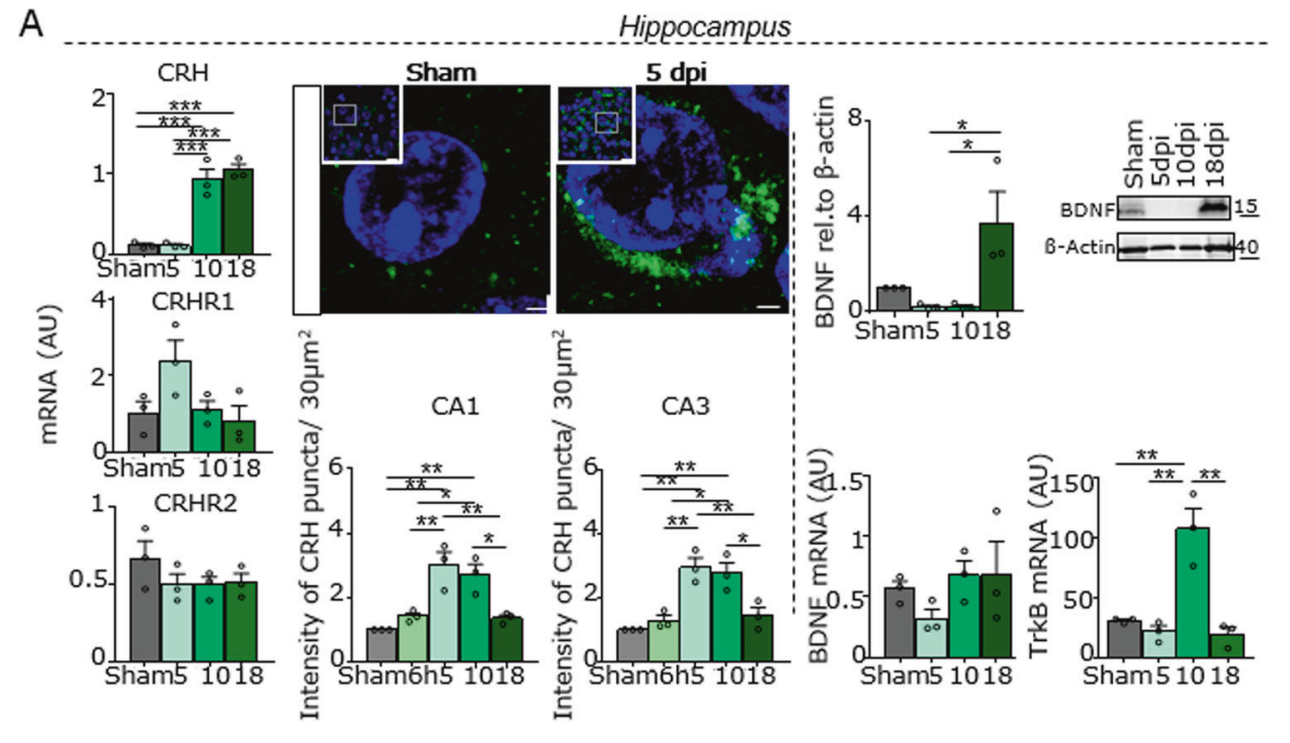

B

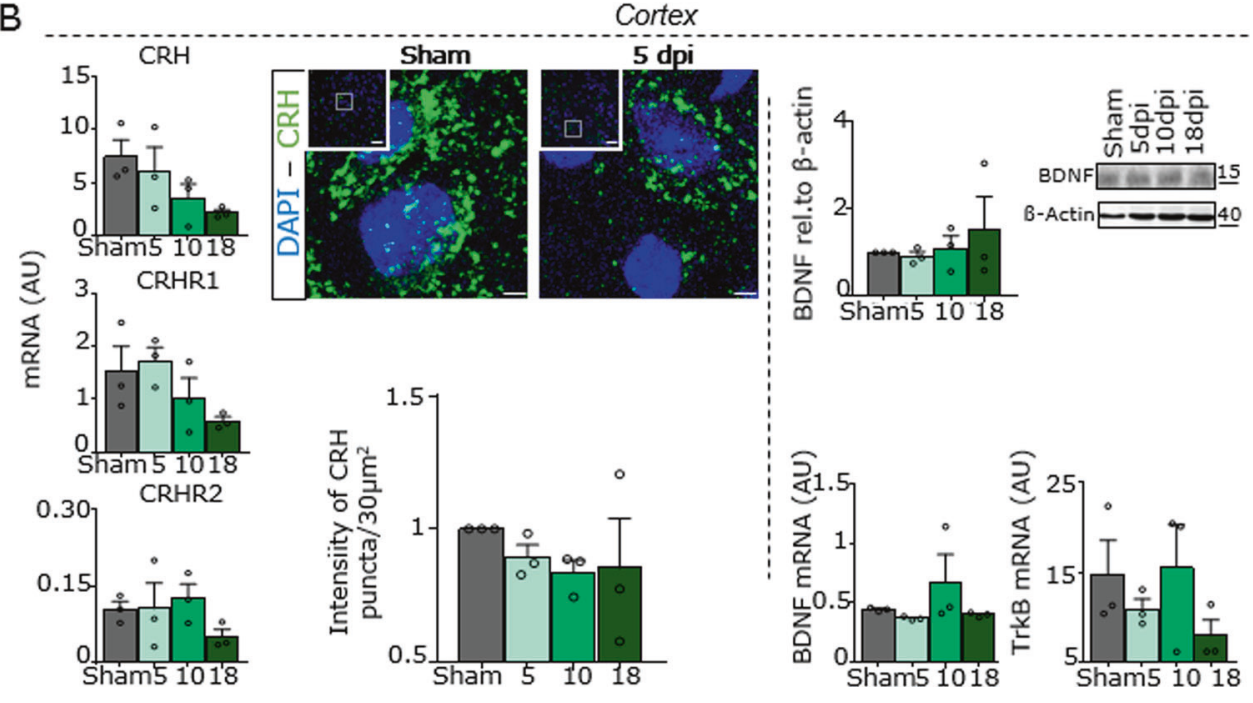

a reduction in BDNF expression. To that end, we closely analyzed proBDNF, BDNF, and BDNF-mRNA levels at different time points after CRH application and found that BDNF mRNA expression was highly upregulated within the first hours, reaching steady state levels after 1 and/or 2 days of incubation. After $30 \mathrm{~min}$, ProBDNF protein levels were unaltered, but the BDNF concentration was significantly downregulated, and it slowly recovered after $5 \mathrm{~h}$ in vitro (Supplementary Fig. 3C-E). Next, we determined the timing of $\mathrm{CRH}$-induced synaptic loss and the concentration required for this effect and tested different concentrations of $\mathrm{CRH}$ ( $30 \mathrm{~min}$ of incubation) as well as different incubation times (with $100 \mathrm{nM}$ CRH) before fixation (Supplementary Fig. 3J, G). We found a significant decrease $(20 \%)$ in the number of excitatory synapses starting at a concentration of $10 \mathrm{nM}$ and observed that $100 \mathrm{nM}$ CRH significantly reduced the number of synaptic contacts after $5 \mathrm{~min}$ (loss of 13\%). BDNF protein levels were clearly reduced after $30 \mathrm{~min}$ of $\mathrm{CRH}$ incubation $(100 \mathrm{nM})$ (Supplementary Fig. 3G). Next, we analyzed the recovery time of hippocampal synapses by adding NBM plus B27 for $30 \mathrm{~min}$ or $5 \mathrm{~h}$ after incubation with $100 \mathrm{nM}$ CRH for $30 \mathrm{~min}$. Both the number of excitatory synapses and the BDNF protein expression were completely restored $5 \mathrm{~h}$ after medium exchange and incubation with neurobasal medium supplemented with B27, L-glutamine (2 mM, Gibco), and 100 units/ml penicillin/streptomycin (Invitrogen) (Supplementary Fig. 3H).

To further elucidate these in vitro findings, we co-applied BDNF as well as specific antagonists for CRHR1 (NBI30775) and corticosterone receptor (RU38486, mifepristone) with CRH (Fig. 3a). First, we found that the additional application of BDNF rescues the loss of synapses in comparison with CRH $(100 \mathrm{nM})$ application (Fig. 3a). Moreover, NBI30775 alone and in combination with mifepristone rescued synaptic loss. Finally, we analyzed 
A

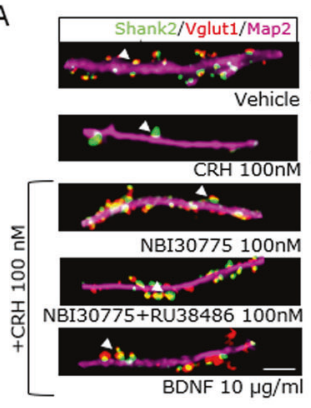

C

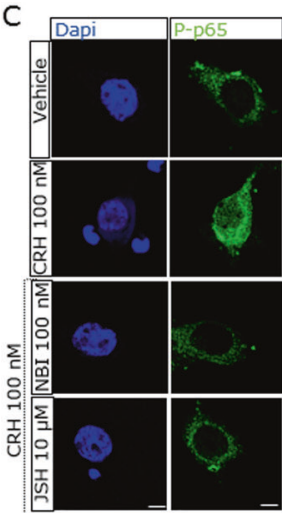

E

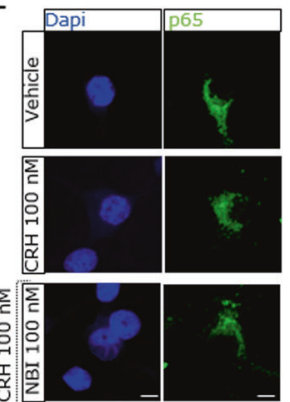

$\mathrm{G}$

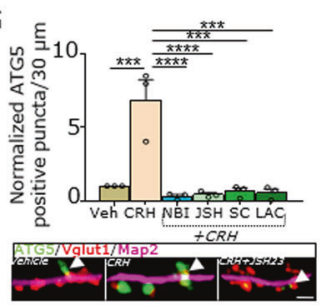

K

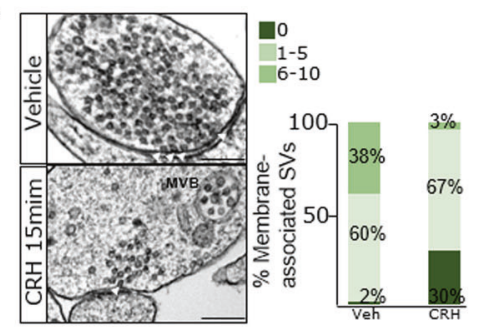

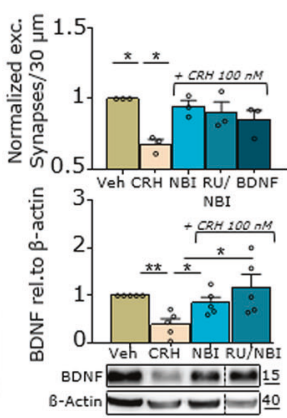
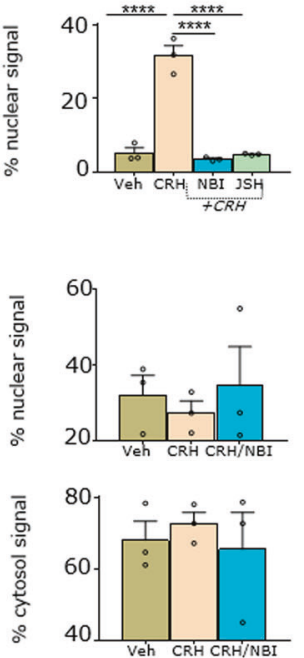

$\mathrm{H}$

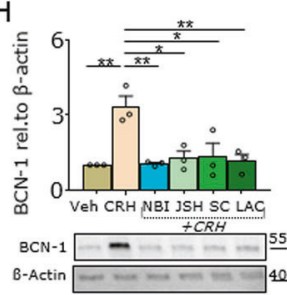

B

D

$\mathrm{F}$
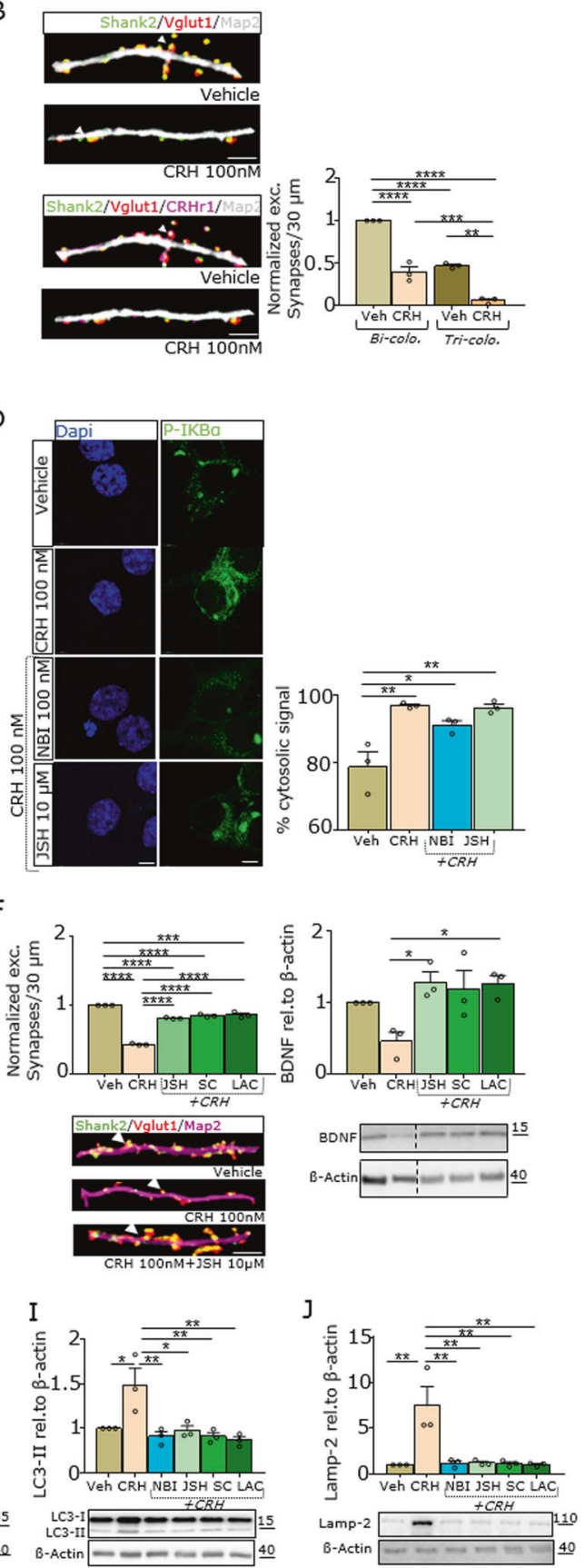

L

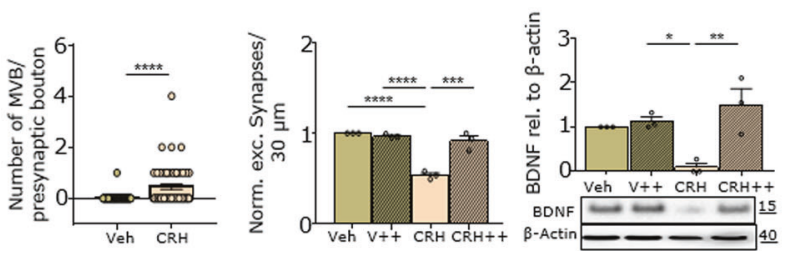

BDNF protein levels under these treatment conditions and observed unaltered BDNF expression after blockage of CRHR1 (Fig. 3a). When we investigated synaptic activity under experimental conditions by the synaptotagmin assay (Supplementary Fig. 3I) and quantified the percentage of active synapses (positive for synaptotagmin-1) in relation to 
Fig. 3 Loss of excitatory synapses in hippocampal cell culture is CRH/BDNF/NF-кB-dependent. a IHC for Shank2 (green), Vglut1 (red), MAP2 (magenta) (scale bar $=5 \mu \mathrm{m}$ ), western blot analysis for all different treatment regimens (CRH; the CRHR1 blocker NBI30775; the corticosterone receptor blocker RU38486; and BDNF) and the relative quantification. b IHC for Shank2 (green), Vglut1 (red), MAP2 (magenta) and CRHR1 (white) with colocalization and trilocalization analysis (scale bar $=5 \mu \mathrm{m}$ ). c IHC for phospho-NF-kB p65 (green, scale $b a r=5 \mu \mathrm{m})$ and the relative quantification of the signal within the nuclear compartment. d IHC for phospho-IKB- $\alpha$ and the relative quantification of the signal within the cytosolic compartment. e IHC for and compartment analysis of p65. f IHC (left panel) for Shank2 (green), Vglut1 (red), MAP2 (magenta) (scale bar $=5 \mu \mathrm{m}$ ), WB of BDNF (right panel) for all different treatment regimens blocking the NF- $\mathrm{BB}$ pathway (JSH (the translocation blocker JSH- 23); SC (the IKK- $\beta$ inhibitor SC-514); and LAC (the NF- $\mathrm{KB}$ activation blocker lactacystin)) and the relative quantification. $\mathbf{g}$ IHC for ATG5, immunoblot analysis of $\mathbf{h} \mathrm{BCN}-1$, $\mathbf{i} \mathrm{LC} 3$, and $\mathbf{j}$ Lamp-2 and the relative quantification normalized to $\beta$-actin. $\mathbf{k}$ TEM of acquired synapses after $15 \mathrm{~min}$ of $\mathrm{CRH}$ treatment (arrows indicate membrane-associated vesicles (scale $b a r=0.5 \mu \mathrm{m})$ ) and the relative quantification of docked vesicles and multivesicular bodies (MVBs)/synapses (a twotailed unpaired $T$-test was used). I Quantification of the number of excitatory synapses (left) by IHC and WB analysis of BDNF expression (right) after treatment with $100 \mathrm{nM} \mathrm{CRH}$ for $30 \mathrm{~min}$ and Leupeptin hemisulfate $+5 \mu \mathrm{M}$ E64 $(\mathrm{CRH}++)$ compared with control treatment. $N=3-5$; the error bars represent the SEMs; one-way ANOVA and Bonferroni's post hoc comparison test were performed $(* P \leq 0.05, * * P \leq 0.005, * * * P \leq 0.0005, * * * * P \leq 0.0001)$.

the total number of excitatory synapses, we did not observe any differences among the different experimental groups, indicating that the activity of the remaining synapses after CRH application remained unchanged. Finally, we investigated the expression of CRHR 1 in individual synapses and found that $\sim 50 \%$ of excitatory synapses were CRHR1 positive. Interestingly, after $\mathrm{CRH}$ treatment, CRHR1positive synapses were no longer detectable (Fig. 3b), indicating that CRHR1-positive synapses are targeted and deleted by $\mathrm{CRH}$.

\section{CRH treatment induces autophagy activation via the NF-KB pathway in hippocampal neurons}

To identify the regulatory action of CRH on BDNF levels, we employed a phospho-protein array that analyzed hippocampal neurons after treatment with CRH $(100 \mathrm{nM})$ versus control. Among 1318 candidates, we found 40 phospho-proteins that were greatly upregulated or downregulated. Interestingly, the expression of NF- $\kappa \mathrm{B}$ p65 itself and a group of $20 \mathrm{NF}-\kappa \mathrm{B}$ pathway-associated proteins was upregulated after CRH treatment (Supplementary Fig. 3M). To confirm these findings, we investigated the expression and localization of phospho-NF- $\mathrm{B}$ p $65, \mathrm{NF}-\kappa \mathrm{B}$ p 65 , and phospho-IKB- $\alpha$ in neurons after CRH application. Moreover, we tested the effects of the CRHR1 blocker NBI30775 and JSH23 (a specific blocker of NF- $\mathrm{B}$ nuclear translocation) on $\mathrm{NF}-\kappa \mathrm{B}$ activation. We found that $\mathrm{CRH}$ treatment increased phospho-NF- $\mathrm{B}$ p65 in the nucleus and elevated phospho-IKB- $\alpha$ levels in the cytosol. Such translocation was not observed after NBI30775 or JSH23 co-application (Fig. 3c, d), and the overall expression of p65 did not change after $\mathrm{CRH}$ administration (Fig. 3e). In addition, we confirmed the ability of $\mathrm{CRH}$ to induce $\mathrm{NF}-\kappa \mathrm{B}$ nuclear translocation via the canonical pathway by applying two additional compounds that blocked the NF- $\kappa \mathrm{B}$ pathway at different steps of activation (SC-514 and lactacystin) (Supplementary Fig. 3L). Finally, we demonstrated that the blockage of the NF- $\mathrm{B}$ pathway in combination with $\mathrm{CRH}$ application maintained BDNF protein expression levels and the number of excitatory synapses (Figs. $3 f$ and 5).

Considering the rapid downregulation of BDNF protein levels, we next analyzed specific cellular degradation mechanisms that are known to be induced by the NF- $\mathrm{BB}$ signaling system, in particular autophagy. To that end, we tested the expression of several autophagy-related factors in vitro and found that ATG5, BCN-1, LC3, and Lamp-2 were strongly upregulated after $30 \mathrm{~min}$ of $\mathrm{CRH}$ treatment, an effect that was abolished by co-application of NF- $\kappa \mathrm{B}$ and CRHR1 blockers (Fig. 3g-j). Moreover, TEM analysis of synaptic contacts revealed a high number of presynaptic terminals with MVBs and fewer membrane-associated vesicles (Fig. 3k and Supplementary Fig. 3M) after $\mathrm{CRH}$ treatment. Finally, we further investigated the role of lysosomal degradation by applying leupeptin hemisulfate (a reversible inhibitor of cysteine, serine, and threonine proteases) and E64 (a protease inhibitor) together with $\mathrm{CRH}$ and found that synapse number and BDNF levels remained unchanged when $\mathrm{CRH}$ treatment was combined with both inhibitors (Fig. 31).

\section{The application of CRHR1 antagonists rescue posttraumatic molecular, structural, and behavioral alterations in old mice in vivo}

In light of age-dependent alterations in synaptic plasticity [33] and the enhanced susceptibility of elderly patients to posttraumatic neuropsychiatric symptoms, we finally investigated the effects of trauma in aged mice compared with younger animals. Comparing the absolute number of hippocampal excitatory synapses between young and older mice showed that old animals had fewer excitatory synapses before trauma. In particular, in the CA3 region, this difference reached $\sim 50 \%$. After the induction of TxT in the older mice, a $50 \%$ reduction in the number of synapses was observed (5 dpi), and this reduction slowly recovered after 18 dpi (Figs. 4a and 5). These data also indicated that after TxT, old mice had only $\sim 20-25 \%$ of the number of hippocampal excitatory synapses that young WT mice had. The concomitant upregulation of hippocampal CRH was also observed to a similar extent in old animals (Fig. 4b). 
A

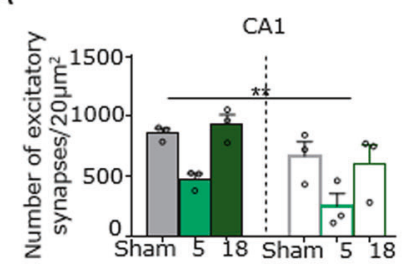

C
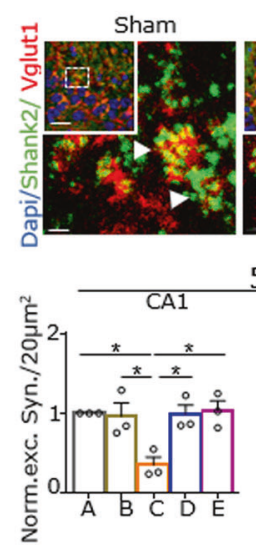

$5 \mathrm{dpi}$
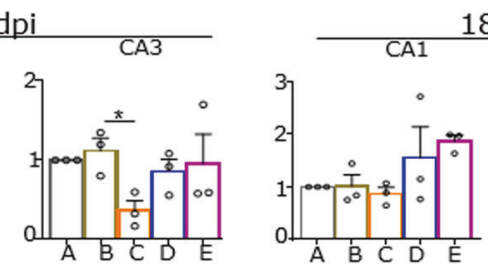

E
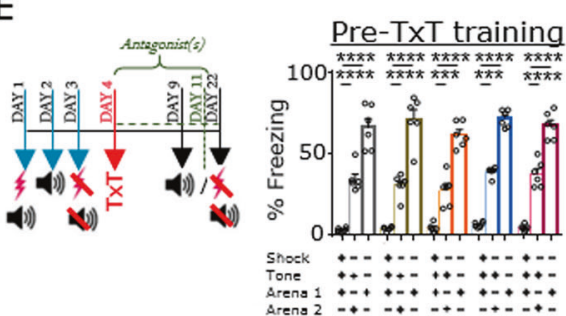

F
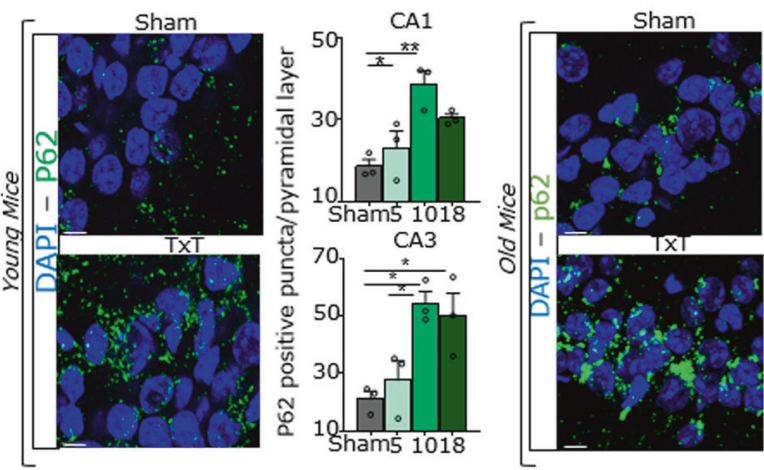

Fig. 4 CRHR1 antagonists rescue autophagy-related hippocampal synaptic loss and memory impairment after thoracic trauma. a Comparison of the number of excitatory synapses in the hippocampal CA1 and CA3 regions of young and old (empty columns) mice (Shank2 and Vglut1 were used as post- and presynaptic markers). b CRH expression (green, scale bar $=20 \mu \mathrm{m}$, magnification scale bar $=5 \mu \mathrm{m}$ ) in the hippocampus of aged mice. $\mathbf{c}$ IHC for Shank2 (green) and Vglut1 (red) and colocalization analysis in the CA1 and CA3 regions of the treated groups (see internal legend) (scale bar $=15$ $\mu \mathrm{m}$, magnification scale $\mathrm{bar}=1 \mu \mathrm{m})$. Arrowheads indicate synaptic colocalization. d Immunoblot of BDNF in the P2 hippocampal fraction
B

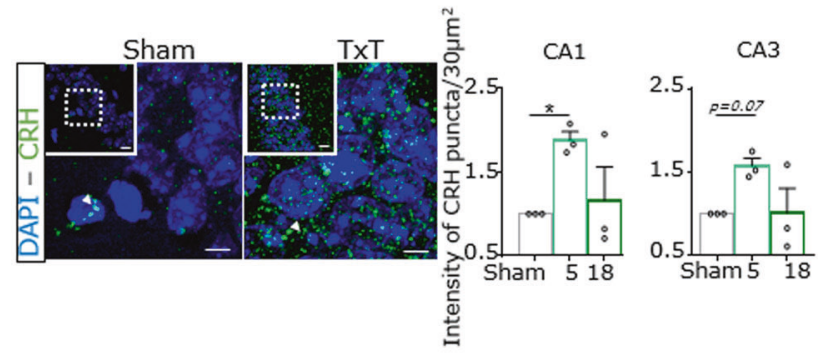

TXT+ NBI30775+
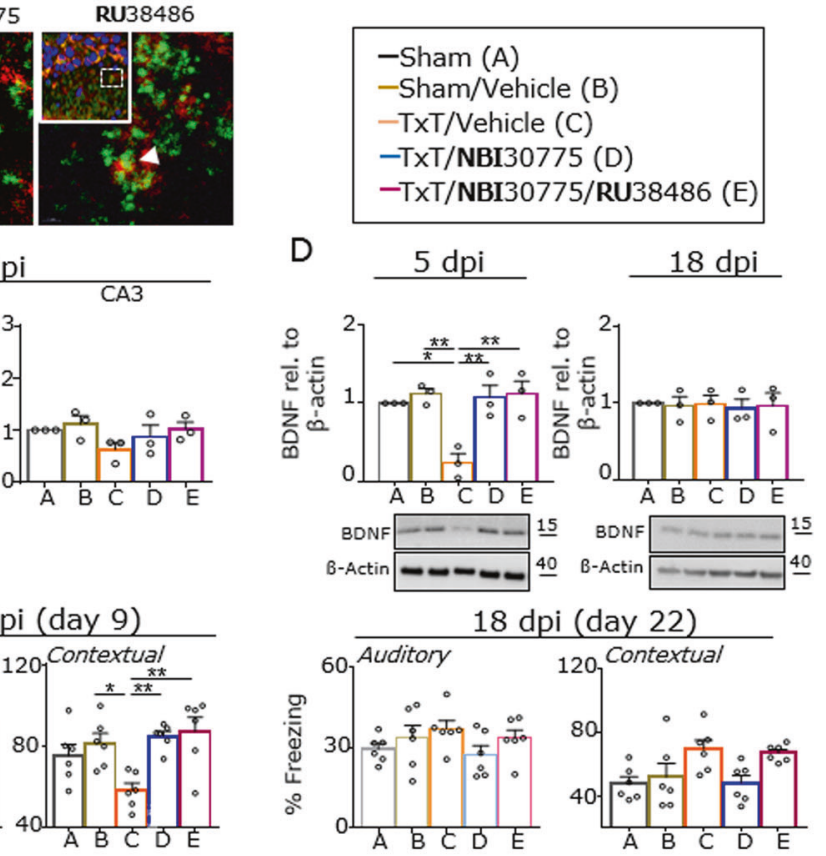

-Sham (A)

-Sham/Vehicle (B)

-TxT/Vehicle (C)

-TxT/NBI30775 (D)

-TxT/NBI30775/RU38486 (E)

D

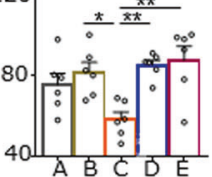

5 dpi (day 9)

- 120 Contextual

G

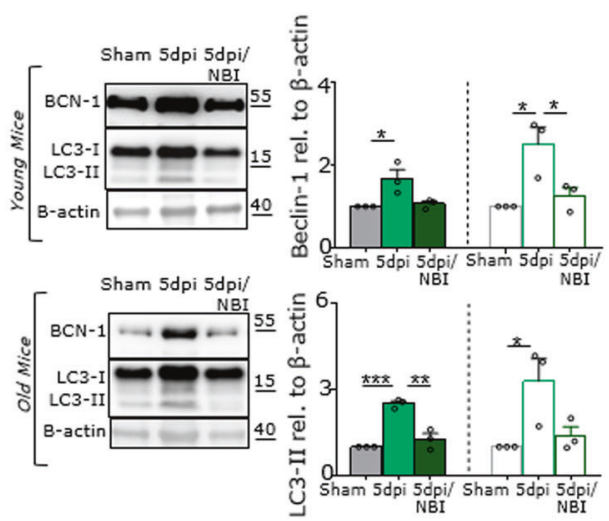

and the relative quantification of the treatment groups. e Schematic of (left to right) the behavioral experiment, analysis of memory and learning before trauma, and analysis of auditory and contextual memory 5 and 18 days after TxT. f IHC for p62 in young (left) and old (right) mice in the hippocampal CA1 and CA3 regions (scale bar $=5 \mu \mathrm{m})$. $\mathrm{g}$ Immunoblot of Beclin-1 and LC3 in hippocampal homogenates from young and old mice 5 days after trauma with or without CRHR1 blockage. $N=3-6$; the error bars represent the SEMs; oneway ANOVA and Bonferroni's post hoc comparison test were always performed $(* P \leq 0.05, * * P \leq 0.005, * * * P \leq 0.0005, * * * * P \leq 0.0001)$. 
Fig. 5 Summary of the main findings of the study. a Thoracic trauma (blue line) induces a selective loss of excitatory synapses in the hippocampus that is caused by CRH expression, leading to local degradation of BDNF. The physiological recovery of these parameters after TxT in mice is observed approximately at day 18 dpi. The application of CRH receptor 1 with or without the co-application of corticosterone receptor antagonists (red line) stabilizes hippocampal BDNF expression. Thereby, the morphological and behavioral alterations are rescued. b A thumbnail sketch of the proposed underlying cellular and molecular mechanisms. Peripheral trauma (here, TxT) induces a pronounced elevation of the hippocampal release of $\mathrm{CRH}$, which activates the canonical NF- $\mathrm{NB}$ pathway via synaptic CRHR1s. This activation induces the autophagy machinery, leading to the local degradation of BDNF and eventually to the loss of excitatory synapses in the hippocampus.

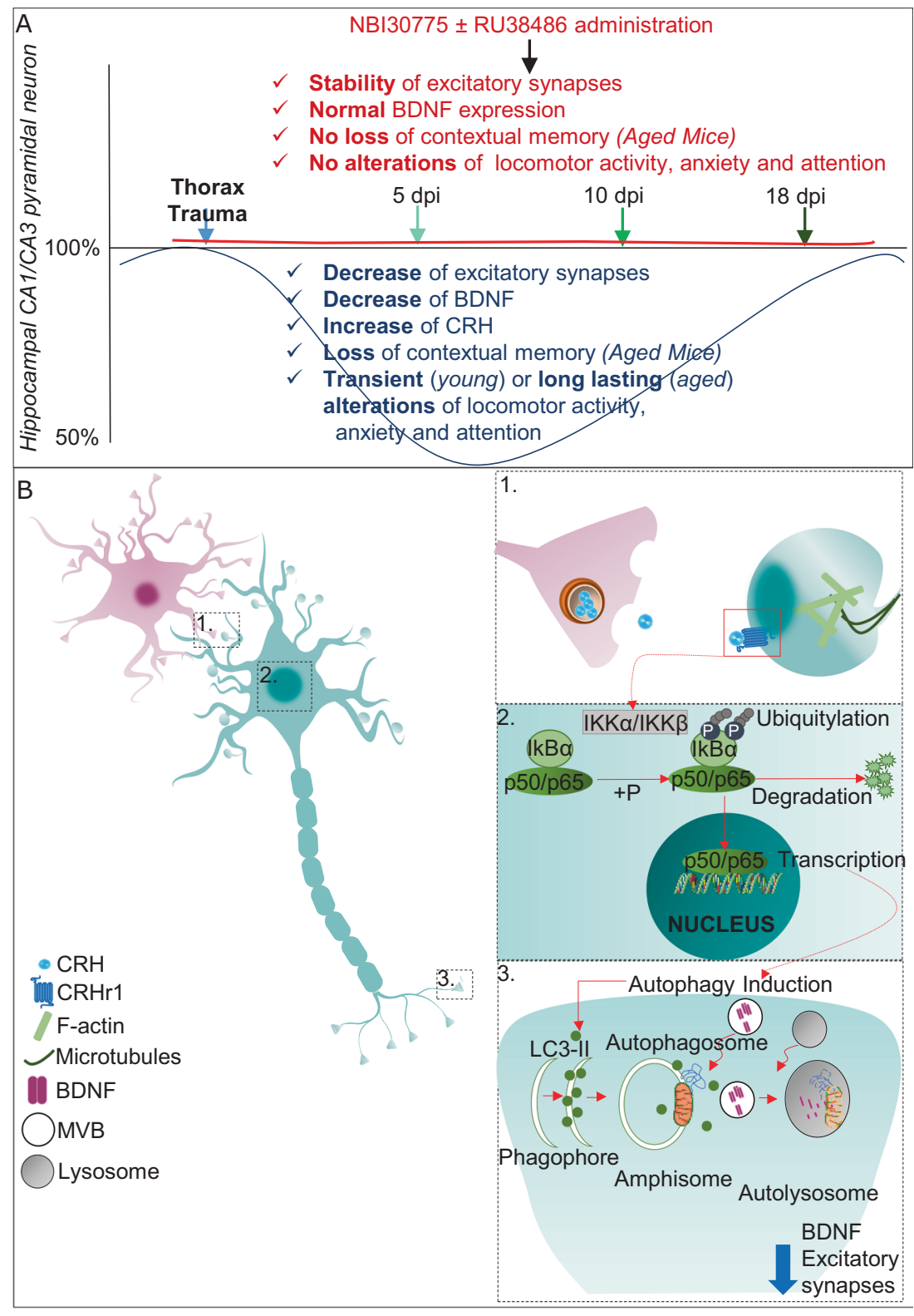

Based on these results and the in vitro results, we next designed an experimental trial to evaluate the therapeutic potential of CRHR1 antagonists (and mifepristone) after trauma in old (Fig. 4) and young animals in vivo (Supplementary Fig. 4). We did not detect a reduction in the number of excitatory synapses in trauma-exposed animals that received the antagonists compared with traumaexposed animals (C), which exhibited 50-60\% synaptic loss at $5 \mathrm{dpi}$. In the trauma-exposed group at $18 \mathrm{dpi}$, synapses naturally recovered so that no differences between the treatment groups were observable when the CA1 and CA3 regions were analyzed (Fig. 4c and Supplementary Fig. 4A). As proof of principle, we also analyzed BDNF protein levels in the hippocampus under these experimental conditions and observed unchanged BDNF levels (Fig. 4d and Supplementary Fig. 4C). Finally, mice were exposed to cue/contextual fear conditioning before trauma and on 5 days and 18 days after trauma (Fig. 4e and Supplementary Fig. 4B). All groups showed an increase in freezing responses during the auditory/contextual memory test in the pre-TxT period. In older mice exposed to trauma and treated with NBI30775 alone or in combination with mifepristone, lesions of the hippocampus appeared to interfere with the acquisition of contextual freezing responses but not cueelicited freezing responses 5 days after trauma (Fig. $4 \mathrm{e}$ and Supplementary Fig. 4B). To further validate our model and to make it comparable with other published delirium models, we expanded the phenotypical characterization of all experimental groups by performing a battery of behavioral tests (the open field test, Y maze, and elevated plus-maze) 
[34-39] on young (Supplementary Fig. 5A-C) and aged mice (Supplementary Fig. 5D-F) $24 \mathrm{~h}$ prior to TxT and $4 \mathrm{~h}$, $24 \mathrm{~h}, 5$ days and 18 days after trauma. We found a strong impairment of locomotor activity, cognitive performance and anxiety behavior in young and old trauma-exposed mice at $4 \mathrm{~h}$ post injury. These alterations quickly recovered in young animals but were still evident in older mice at 24 hpi and 5 days after trauma. Interestingly, the administration of NBI30775 alone or in combination with mifepristone reversed nearly all the observed behavioral alterations after trauma in young and aged animals.

Finally, we analyzed the involvement of autophagy in posttraumatic synaptic loss under in vivo conditions and used antibodies directed against p62, BCN-1, and LC3 (Fig. 4f, g). Regarding p62, we found an increase in p62-positive puncta in the hippocampi of younger mice, particularly after 10 days. In older mice, we observed the same trend at $5 \mathrm{dpi}$. The hippocampal protein expression of BCN-1 and LC3 after trauma was found to be significantly upregulated in younger and aged mice at $5 \mathrm{dpi}$; this effect was abrogated by NBI30775 administration (Fig. 4g).

\section{Discussion}

Physical trauma or complicated, long-lasting surgical operations are closely associated with neuropsychiatric syndromes that are subsumed as acute physical stress response/disorder or delirium [40, 41]. In industrial countries, delirium affects $\sim 15 \%$ of all inpatients $[42,43]$ and is highly correlated with higher mortality and extended hospital stays [44]. Despite the great efforts of scientists and clinicians to unravel the pathophysiology of delirium and to develop effective treatment options, this acute and mostly transient neuropsychiatric disorder is still far from being completely understood. This is also because the construct, face and predictive validity of animal models of delirium is very limited due to the multiple causes and broad spectrum and variety of symptoms. In one of the first rodent delirium models, the muscarinic receptor antagonist atropine was administered and led to acute cognitive deficits in a blind alley maze and EEG slowing reminiscent of delirium [45]. Animals with selective lesions of the basal forebrain cholinergic system have been shown to be susceptible to acute cognitive dysfunction that is reversible upon inflammatory resolution [31]. In fact, more recently, several delirium models based on the induction of an inflammatory response were introduced. Delirium-like behavioral alterations are observed after the application of high doses of lipopolysaccharide (LPS) or cecal ligation and puncture leading to polymicrobial sepsis [46]. For example, Chen et al. [47] showed that compared with younger mice, old mice exhibit an increased inflammatory response in the hippocampus after LPS challenge, and it was found that hippocampal processing is more easily disrupted in old animals than in younger ones. In line with these findings, in ME7 animals (a mouse model of prion disease), the application of LPS induces acute and transient working memory deficits, and ME7 animals show heightened and prolonged transcription of inflammatory mediators in the CNS [48].

In this study, we wanted to mimic postoperative [39] and posttraumatic cognitive dysfunction without primarily triggering inflammatory responses. To that end, we induced peripheral trauma (TxT) to analyze the molecular, cellular, and behavioral features of delirium. Interestingly, in this new rodent model, we observed a broad spectrum of behavioral deficits (especially in aged animals) reminiscent of delirium. We found that TxT has a significant impact on the homeostasis of hippocampal excitatory synapses. In aged animals, the transient $50 \%$ synapse reduction in the hippocampus resulted in an impairment of memory formation (Fig. 5). The loss of synapses may have been attributable to the autophagic degradation of BDNF that induced by local hippocampal release of CRH through the CRHR1/ NF- $\mathrm{kB}$ pathway. Blocking CRHR1 rescued delirium-like symptoms in vivo. Our data suggest that the pathophysiological basis of the rapid decline in cognitive function might indeed be explained by synaptic loss and subsequent functional impairment of local circuitries within the hippocampal formation [19].

To exclude other putative mechanisms/factors that are known to regulate synapse number, we closely analyzed neuronal loss, hypoxia and complement cascade involvement $[49,50]$ as well as severe signs of neuroinflammation. As mentioned before, these mechanisms have also been proposed to cause delirium (mainly based on clinical data sets [4]). We especially focused on microglial and astroglial activation [51-53] but we were unable to detect specific signs of inflammatory responses after TxT.

Interestingly, the synaptic alterations that were found were brain region- and neuron/synapse-specific, indicating a selective vulnerability of the hippocampus to peripheral trauma. This regional specificity was most likely due to the local expression of $\mathrm{CRH}$ in hippocampal interneurons, as in vitro data indicated that cortical neurons were CRH-responsive in principle. Moreover, our experiments on primary hippocampal neurons indicated that synapses involving the dendritic trees of pyramidal neurons expressing CRHR1 were specifically deleted. Of note, the remaining excitatory synapses showed no morphological differences compared with synapses under sham conditions and showed no differences in functional properties in the synaptotagmin assay.

It is well known that stress influences memory by modulating the integrity and plasticity of synapses, which are fundamental for memory processes [54-57]. Here, we 
propose a novel mechanistic concept of the development of delirium based on the idea of hippocampal stress response [12, 58-61] triggered by an elevation of posttraumatic interneuronal CRH production and release [62].

There are important published data on the molecular mechanisms by which a reduction in the number of spines and synapses is induced by CRH release. The authors proposed that CRH-CRHR1-mediated activation of a RhoGTPase induces actin depolymerization and subsequent spine loss [61, 63]. Based on these data, we screened for additional potential mediators of the action of $\mathrm{CRH}$ on pyramidal neurons and found that BDNF [15], a prominent signaling molecule that influences synaptogenesis and spine formation [64], neuronal survival [65], LTP, neuronal excitability [66], and adult hippocampal neurogenesis [67], was almost entirely depleted in the hippocampus after TxT.

Eventually, we identified a novel pathway by which $\mathrm{CRH}$ exerts its effect on BDNF and found that the NF- $\mathrm{KB}$ signaling cascade is essentially involved. To our knowledge, this is the first study that identifies a CRH-CRHR1NF- $\mathrm{\kappa B}$ interaction in primary neurons (Fig. 5). However, it has already been shown that CRH specifically activates the NF- $\kappa B$ pathway via CRHR1 in thymocytes $[68,69]$. Furthermore, it has been shown that the activated NF- $\mathrm{KB}$ pathway can induce autophagy by upregulating $\mathrm{BCN}-1$ [70]. Indeed, we observed in our experimental setup that BCN-1, LC3, and Lamp-2 were significantly upregulated by elevated CRH levels in neurons (in vitro and in vivo) and that signs of autophagy induction, especially in the presynaptic compartment, appeared. Interestingly, recent evidence of fine-tuned protein homeostasis regulated by autophagy at synapses, especially in the presynaptic compartment, was reported [71-73]. We found that CRHR1 activation was correlated with the rapid downregulation of (presynaptic) BDNF and that BDNF degradation was rescued by CRHR1 blockage as well as NF- $\mathrm{kB}$ pathway inhibition. Finally, we tested the hypothesis that elevated $\mathrm{CRH}$ levels induce rapid lysosomal/autophagic BDNF degradation in hippocampal neurons [74] and applied proteosomal inhibitors. We thereby stabilized BDNF levels as well as synapse integrity in vitro, supporting the novel concept of a direct regulatory link between CRH and BDNF. The loss of synapses is therefore explained by the local disruption of a proposed BDNF autocrine feedforward loop [75].

As already mentioned before, age is one of the strongest predictors of the development of delirium after trauma, and aged-related neurodegeneration has been discussed as an additional predisposing factor ("second hit") for the development of delirium $[48,76]$. When we compared young and old animals, we did not observe significant differences in the stress reaction in the brain per se; however, the number of hippocampal synapses was already reduced to $\sim 50 \%$ before the traumatic event in the aged mice. This might explain why behavioral deficits after trauma are more severe in elderly patients; it appears much more likely that the total number of synaptic connections falls below a certain threshold, guaranteeing hippocampal functionality. By applying CRHR1 antagonists, however, we successfully rescued synapse numbers and reversed all accompanying histological, molecular and behavioral changes observed after trauma in young and aged animals. The results are also in very good accordance with data obtained from CRHR1 KO mice, which have fewer hippocampal spines than control animals and do not exhibit downregulated spines/synapses in response to early life stress [77].

Based on our results, a posttraumatic delirium-like status might be prevented by inhibiting the CRH-CRHR1-NF-KBBDNF pathway. Several antagonists of the CRH receptor, the best known of which are the selective CRHR1 antagonist Antalarmin and a newer drug pexacerfont, have been developed and are widely used in research. A recent human trial found that pexacerfont was not effective in alleviating the symptoms of general anxiety disorder [78], although additional research is still needed. In monkeys, Antalarmin has been shown to be successful in lowering the stressinduced CRF rise in CSF, suppressing anxiety-associated behaviors, and increased exploratory behavior in stressful situations, but human trials are necessary to test the clinical efficacy of Antalarmin [79-82]. In our study, we applied the CRHR1 antagonist NBI30775, which has already been used in clinical trials for depression/anxiety and has shown good efficacy and safety $[83,84]$. Moreover, there are trials using various compounds that block the NF-kB pathway, especially for the treatment of cancer, that might also benefit after trauma events [85].

In summary, our study provides a novel model of acute physical stress responses and delirium as well as molecular insights into posttraumatic (especially synaptic) changes in the brain that are initiated by the elevation of hippocampal CRH levels (Fig. 5). The therapeutic interruption of this novel trauma-induced signaling cascade might therefore prevent synaptic loss and eventually the occurrence of delirium and reduce its severity.

\section{Data availability}

The authors confirm that the data supporting the findings of this study are available within the article [and/or] its supplementary materials.

Acknowledgements The authors are thankful to Ursula Pika-Hartlaub, Maja Manz, and Nathalie Damm for technical support. The authors are also thankful to Paul Walther and Renate Kunz for TEM support. Open access funding provided by Projekt DEAL. 
Author contributions TMB, together with PR, FR, and MH-L, designed and outlined the study. SC, CRB, CU-R, and SG performed the experiments with help from MS, JB, and SB. SC and CRB analyzed the data with the support of FR, MH-L, and TMB. SC and TMB wrote the manuscript.

Funding This study was supported by the Deutsche Forschungsgemeinschaft (DFG, German Research Foundation)-Projektnummer 251293561-SFB 1149 (project A2) to TMB. TMB was further supported by the BIU2 initiative, the Else Kröner Foundation, the Innovative Medicines Initiative (IMI) Joint Undertaking under grant agreement n777394 (AIMS 2 Trials), which is composed of financial contributions from the European Union and EFPIA companies' in-kind contribution and the DZNE, Ulm site.

\section{Compliance with ethical standards}

Conflict of interest The authors declare that they have no conflict of interest.

Publisher's note Springer Nature remains neutral with regard to jurisdictional claims in published maps and institutional affiliations.

Open Access This article is licensed under a Creative Commons Attribution 4.0 International License, which permits use, sharing, adaptation, distribution and reproduction in any medium or format, as long as you give appropriate credit to the original author(s) and the source, provide a link to the Creative Commons license, and indicate if changes were made. The images or other third party material in this article are included in the article's Creative Commons license, unless indicated otherwise in a credit line to the material. If material is not included in the article's Creative Commons license and your intended use is not permitted by statutory regulation or exceeds the permitted use, you will need to obtain permission directly from the copyright holder. To view a copy of this license, visit http://creativecommons. org/licenses/by/4.0/

\section{References}

1. Griffiths R, Beech F, Brown A, Dhesi J, Foo I, Goodall J, et al. Peri-operative care of the elderly 2014: association of anaesthetists of Great Britain and Ireland. Anaesthesia. 2014;69(Suppl 1) :81-98.

2. Ryan DJ, O'Regan NA, Caoimh RO, Clare J, O'Connor M, Leonard M, et al. Delirium in an adult acute hospital population: predictors, prevalence and detection. BMJ Open. 2013;3:e01772.

3. Kat MG, de Jonghe JF, Vreeswijk R, van der Ploeg T, van Gool WA, Eikelenboom P, et al. Mortality associated with delirium after hip-surgery: a 2-year follow-up study. Age Ageing. 2011; 40:312-8

4. Cerejeira J, Firmino H, Vaz-Serra A, Mukaetova-Ladinska EB. The neuroinflammatory hypothesis of delirium. Acta Neuropathol. 2010;119:737-54.

5. Maclullich AM, Anand A, Davis DH, Jackson T, Barugh AJ, Hall $\mathrm{RJ}$, et al. New horizons in the pathogenesis, assessment and management of delirium. Age Ageing. 2013;42:667-74.

6. Martins S, Fernandes L. Delirium in elderly people: a review. Front Neurol. 2012;3:101.

7. Angles EM, Robinson TN, Biffl WL, Johnson J, Moss M, Tran $\mathrm{ZV}$, et al. Risk factors for delirium after major trauma. Am J Surg. 2008;196:864-9.

8. Zaal IJ, Devlin JW, Peelen LM, Slooter AJ. A systematic review of risk factors for delirium in the ICU. Crit Care Med. 2015;43:40-47.
9. Saxena S, Lawley D. Delirium in the elderly: a clinical review. Postgrad Med J. 2009;85:405-13.

10. Oberai T, Laver K, Crotty M, Killington M, Jaarsma R. Effectiveness of multicomponent interventions on incidence of delirium in hospitalized older patients with hip fracture: a systematic review. Int Psychogeriatr. 2018;30:481-92.

11. Goswami S, Rodriguez-Sierra O, Cascardi M, Pare D. Animal models of post-traumatic stress disorder: face validity. Front Neurosci. 2013;7:89.

12. Chen Y, Dube CM, Rice CJ, Baram TZ. Rapid loss of dendritic spines after stress involves derangement of spine dynamics by corticotropin-releasing hormone. J Neurosci. 2008;28:2903-11.

13. Maras PM, Baram TZ. Sculpting the hippocampus from within: stress, spines, and CRH. Trends Neurosci. 2012;35:315-24.

14. Andres AL, Regev L, Phi L, Seese RR, Chen Y, Gall CM, et al. NMDA receptor activation and calpain contribute to disruption of dendritic spines by the stress neuropeptide CRH. J Neurosci. 2013;33:16945-60.

15. Poo MM. Neurotrophins as synaptic modulators. Nat Rev Neurosci. 2001;2:24-32.

16. Park H, Poo MM. Neurotrophin regulation of neural circuit development and function. Nat Rev Neurosci. 2013;14:7-23.

17. Bennett MR, Lagopoulos J. Stress and trauma: BDNF control of dendritic-spine formation and regression. Prog Neurobiol. 2014;112:80-99.

18. Suri D, Vaidya VA. Glucocorticoid regulation of brain-derived neurotrophic factor: relevance to hippocampal structural and functional plasticity. Neuroscience. 2013;239:196-213.

19. Jarquin-Valdivia AA, Major RJ. A unifying hypothesis for delirium and hospital-acquired weakness as synaptic dysfunctions. Med Hypotheses. 2019;124:105-9.

20. Ehrnthaller C, Flierl M, Perl M, Denk S, Unnewehr H, Ward PA, et al. The molecular fingerprint of lung inflammation after blunt chest trauma. Eur J Med Res. 2015;20:70.

21. Bockers TM, Winter C, Smalla K-H, Kreutz MR, Bockmann J, Seidenbecher C, et al. Proline-rich synapse-associated protein-1/ cortactin binding protein 1 (ProSAP1/CortBP1) is a PDZ-domain protein highly enriched in the postsynaptic density. J Neurosci. 1999;19:6506-18.

22. Bockmann J, Kreutz MR, Gundelfinger ED, Bockers TM. ProSAP/ Shank postsynaptic density proteins interact with insulin receptor tyrosine kinase substrate IRSp53. J Neurochem. 2002;83:1013-7.

23. Schmeisser MJ, Ey E, Wegener S, Bockmann J, Stempel AV, Kuebler A, et al. Autistic-like behaviours and hyperactivity in mice lacking ProSAP1/Shank2. Nature. 2012;486:256-60.

24. Heise C, Schroeder JC, Schoen M, Halbedl S, Reim D, Woelfle S, et al. Selective localization of shanks to VGLUT1-positive excitatory synapses in the mouse hippocampus. Front Cell Neurosci. 2016;10:106.

25. Reim D, Distler U, Halbedl S, Verpelli C, Sala C, Bockmann J, et al. Proteomic analysis of post-synaptic density fractions from Shank3 mutant mice reveals brain region specific changes relevant to autism spectrum disorder. Front Mol Neurosci. 2017;10:26.

26. Villinger C, Schauflinger M, Gregorius H, Kranz C, Hohn K, Nafeey S, et al. Three-dimensional imaging of adherent cells using FIB/SEM and STEM. Methods Mol Biol. 2014;1117:617-38.

27. Von Bartheld CS, Altick AL. Multivesicular bodies in neurons: distribution, protein content, and trafficking functions. Prog Neurobiol. 2011;93:313-40.

28. Schoen M, Reichel JM, Demestre M, Putz S, Deshpande D, Proepper C, et al. Super-resolution microscopy reveals presynaptic localization of the ALS/FTD related protein FUS in hippocampal neurons. Front Cell Neurosci. 2015;9:496.

29. Fanselow MS. Contextual fear, gestalt memories, and the hippocampus. Behav Brain Res. 2000;110:73-81. 
30. Blanchard RJ, Blanchard DC. Crouching as an index of fear. J Comp Physiol Psychol. 1969;67:370-5.

31. Field RH, Gossen A, Cunningham C. Prior pathology in the basal forebrain cholinergic system predisposes to inflammation-induced working memory deficits: reconciling inflammatory and cholinergic hypotheses of delirium. J Neurosci. 2012;32:6288-94.

32. Smith MA, Makino S, Kvetnansky R, Post RM. Stress and glucocorticoids affect the expression of brain-derived neurotrophic factor and neurotrophin-3 mRNAs in the hippocampus. J Neurosci. 1995;15:1768-77.

33. Morrison JH, Baxter MG. The ageing cortical synapse: hallmarks and implications for cognitive decline. Nat Rev Neurosci. 2012;13:240-50.

34. Walf AA, Frye CA. The use of the elevated plus maze as an assay of anxiety-related behavior in rodents. Nat Protoc. 2007;2:322-8.

35. Kraeuter AK, Guest PC, Sarnyai Z. The Y-Maze for assessment of spatial working and reference memory in mice. Methods Mol Biol. 2019;1916:105-11.

36. Felton LM, Cunningham C, Rankine EL, Waters S, Boche D, Perry VH. MCP-1 and murine prion disease: separation of early behavioural dysfunction from overt clinical disease. Neurobiol Dis. 2005;20:283-95.

37. Cunningham C, Deacon R, Wells H, Boche D, Waters S, Diniz $\mathrm{CP}$, et al. Synaptic changes characterize early behavioural signs in the ME7 model of murine prion disease. Eur $\mathrm{J}$ Neurosci. 2003;17:2147-55.

38. Deacon RM, Cholerton LL, Talbot K, Nair-Roberts RG, Sanderson DJ, Romberg C, et al. Age-dependent and -independent behavioral deficits in Tg2576 mice. Behav Brain Res. 2008;189:126-38.

39. Peng M, Zhang C, Dong Y, Zhang Y, Nakazawa H, Kaneki M, et al. Battery of behavioral tests in mice to study postoperative delirium. Sci Rep. 2016;6:29874.

40. Wiseman T, Foster K, Curtis K. Mental health following traumatic physical injury: an integrative literature review. Injury. 2013;44:1383-90.

41. Karnatovskaia LV, Johnson MM, Benzo RP, Gajic O. The spectrum of psychocognitive morbidity in the critically ill: a review of the literature and call for improvement. J Crit Care. 2015;30:130-7.

42. Jackson TA, Gladman JR, Harwood RH, MacLullich AM, Sampson EL, Sheehan B, et al. Challenges and opportunities in understanding dementia and delirium in the acute hospital. PLoS Med. 2017;14:e1002247.

43. Davis D, Richardson S, Hornby J, Bowden H, Hoffmann K, Weston-Clarke $\mathbf{M}$, et al. The delirium and population health informatics cohort study protocol: ascertaining the determinants and outcomes from delirium in a whole population. BMC Geriatr. 2018; $18: 45$

44. Dani M, Owen LH, Jackson TA, Rockwood K, Sampson EL, Davis D. Delirium, frailty, and mortality: interactions in a prospective study of hospitalized older people. J Gerontol A Biol Sci Med Sci. 2018;73:415-8.

45. Leavitt ML, Trzepacz PT, Ciongoli K. Rat model of delirium: atropine dose-response relationships. J Neuropsychiatry Clin Neurosci. 1994;6:279-84.

46. Semmler A, Hermann S, Mormann F, Weberpals M, Paxian SA, Okulla T, et al. Sepsis causes neuroinflammation and concomitant decrease of cerebral metabolism. J Neuroinflammation. 2008;5:38.

47. Chen J, Buchanan JB, Sparkman NL, Godbout JP, Freund GG, Johnson RW. Neuroinflammation and disruption in working memory in aged mice after acute stimulation of the peripheral innate immune system. Brain Behav Immun. 2008;22:301-11.

48. Murray C, Sanderson DJ, Barkus C, Deacon RM, Rawlins JN, Bannerman DM, et al. Systemic inflammation induces acute working memory deficits in the primed brain: relevance for delirium. Neurobiol Aging. 2012;33:603-.e3.
49. Vasek MJ, Garber C, Dorsey D, Durrant DM, Bollman B, Soung A, et al. A complement-microglial axis drives synapse loss during virus-induced memory impairment. Nature. 2016;534:538-43.

50. Segura I, Lange C, Knevels E, Moskalyuk A, Pulizzi R, Eelen G, et al. The oxygen sensor PHD2 controls dendritic spines and synapses via modification of filamin A. Cell Rep. 2016;14:2653-67.

51. Fan Z, Brooks DJ, Okello A, Edison P. An early and late peak in microglial activation in Alzheimer's disease trajectory. Brain. 2017;140:792-803.

52. Belanger M, Magistretti PJ. The role of astroglia in neuroprotection. Dialogues Clin Neurosci. 2009;11:281-95.

53. Hong S, Beja-Glasser VF, Nfonoyim BM, Frouin A, Li S, Ramakrishnan S, et al. Complement and microglia mediate early synapse loss in Alzheimer mouse models. Science. 2016;352:712-6.

54. Kim JJ, Diamond DM. The stressed hippocampus, synaptic plasticity and lost memories. Nat Rev Neurosci. 2002;3:453-62.

55. Maggio N, Segal M. Differential corticosteroid modulation of inhibitory synaptic currents in the dorsal and ventral hippocampus. J Neurosci. 2009;29:2857-66.

56. Popoli M, Yan Z, McEwen BS, Sanacora G. The stressed synapse: the impact of stress and glucocorticoids on glutamate transmission. Nat Rev Neurosci. 2011;13:22-37.

57. Howland JG, Wang YT. Synaptic plasticity in learning and memory: stress effects in the hippocampus. Prog Brain Res. 2008;169:145-58.

58. Donohue HS, Gabbott PL, Davies HA, Rodriguez JJ, Cordero MI, Sandi C, et al. Chronic restraint stress induces changes in synapse morphology in stratum lacunosum-moleculare CA1 rat hippocampus: a stereological and three-dimensional ultrastructural study. Neuroscience. 2006;140:597-606.

59. Pawlak R, Rao BS, Melchor JP, Chattarji S, McEwen B, Strickland $\mathrm{S}$. Tissue plasminogen activator and plasminogen mediate stressinduced decline of neuronal and cognitive functions in the mouse hippocampus. Proc Natl Acad Sci USA. 2005;102:18201-6.

60. Chen Y, Rex CS, Rice CJ, Dube CM, Gall CM, Lynch G, et al. Correlated memory defects and hippocampal dendritic spine loss after acute stress involve corticotropin-releasing hormone signaling. Proc Natl Acad Sci USA. 2010;107:13123-8.

61. Chen Y, Andres AL, Frotscher M, Baram TZ. Tuning synaptic transmission in the hippocampus by stress: the CRH system. Front Cell Neurosci. 2012;6:13.

62. Hooper A, Maguire J. Characterization of a novel subtype of hippocampal interneurons that express corticotropin-releasing hormone. Hippocampus. 2016;26:41-53.

63. Zhao JY, Song B, Anand E, Schwartz D, Panesar M, Jackson GP, et al. Barriers, facilitators, and solutions to optimal patient portal and personal health record use: a systematic review of the literature. AMIA Annu Symp Proc. 2017;2017:1913-22.

64. Yoshii A, Constantine-Paton M. Postsynaptic BDNF-TrkB signaling in synapse maturation, plasticity, and disease. Dev Neurobiol. 2010;70:304-22.

65. Lipsky RH, Marini AM. Brain-derived neurotrophic factor in neuronal survival and behavior-related plasticity. Ann N Y Acad Sci. 2007;1122:130-43.

66. Minichiello L. TrkB signalling pathways in LTP and learning. Nat Rev Neurosci. 2009;10:850-60.

67. Schmidt HD, Duman RS. The role of neurotrophic factors in adult hippocampal neurogenesis, antidepressant treatments and animal models of depressive-like behavior. Behav Pharm. 2007;18:391-418.

68. Zhao J, Karalis KP. Regulation of nuclear factor-kappaB by corticotropin-releasing hormone in mouse thymocytes. Mol Endocrinol. 2002;16:2561-70.

69. Grammatopoulos DK. Insights into mechanisms of corticotropinreleasing hormone receptor signal transduction. $\mathrm{Br} \mathrm{J}$ Pharm. 2012;166:85-97. 
70. Trocoli A, Djavaheri-Mergny M. The complex interplay between autophagy and NF-kappaB signaling pathways in cancer cells. Am J Cancer Res. 2011;1:629-49.

71. Okerlund ND, Schneider K, Leal-Ortiz S, Montenegro-Venegas C, Kim SA, Garner LC, et al. Bassoon controls presynaptic autophagy through Atg5. Neuron. 2017;93:897-913 e897.

72. Hoffmann S, Orlando M, Andrzejak E, Bruns C, Trimbuch T, Rosenmund $\mathrm{C}$, et al. Light-activated ROS production induces synaptic autophagy. J Neurosci. 2019;39:2163-83.

73. Vijayan V, Verstreken P. Autophagy in the presynaptic compartment in health and disease. J Cell Biol. 2017;216:1895-906.

74. Evans SF, Irmady K, Ostrow K, Kim T, Nykjaer A, Saftig P, et al. Neuronal brain-derived neurotrophic factor is synthesized in excess, with levels regulated by sortilin-mediated trafficking and lysosomal degradation. J Biol Chem. 2011;286:29556-67.

75. Sampathkumar C, Wu YJ, Vadhvani M, Trimbuch T, Eickholt B, Rosenmund C. Loss of MeCP2 disrupts cell autonomous and autocrine BDNF signaling in mouse glutamatergic neurons. Elife. 2016;5:e19374.

76. Skelly DT, Griffin EW, Murray CL, Harney S, O'Boyle C, Hennessy E, et al. Acute transient cognitive dysfunction and acute brain injury induced by systemic inflammation occur by dissociable IL-1dependent mechanisms. Mol Psychiatry. 2019;24:1533-48.

77. Wang XD, Chen Y, Wolf M, Wagner KV, Liebl C, Scharf SH, et al. Forebrain CRHR1 deficiency attenuates chronic stressinduced cognitive deficits and dendritic remodeling. Neurobiol Dis. 2011;42:300-10.

78. Coric V, Feldman HH, Oren DA, Shekhar A, Pultz J, Dockens RC, et al. Multicenter, randomized, double-blind, active comparator and placebo-controlled trial of a corticotropin-releasing factor receptor-1 antagonist in generalized anxiety disorder. Depress Anxiety. 2010; 27:417-25.
79. Habib KE, Weld KP, Rice KC, Pushkas J, Champoux M, Listwak S, et al. Oral administration of a corticotropin-releasing hormone receptor antagonist significantly attenuates behavioral, neuroendocrine, and autonomic responses to stress in primates. Proc Natl Acad Sci USA. 2000;97:6079-84.

80. Deak T, Nguyen KT, Ehrlich AL, Watkins LR, Spencer RL, Maier SF, et al. The impact of the nonpeptide corticotropinreleasing hormone antagonist antalarmin on behavioral and endocrine responses to stress. Endocrinology. 1999;140: 79-86.

81. Wong ML, Webster EL, Spokes H, Phu P, Ehrhart-Bornstein M, Bornstein S, et al. Chronic administration of the non-peptide CRH type 1 receptor antagonist antalarmin does not blunt hypothalamic-pituitary-adrenal axis responses to acute immobilization stress. Life Sci. 1999;65:PL53-8.

82. Bornstein SR, Webster EL, Torpy DJ, Richman SJ, Mitsiades N, Igel $\mathrm{M}$, et al. Chronic effects of a nonpeptide corticotropinreleasing hormone type I receptor antagonist on pituitary-adrenal function, body weight, and metabolic regulation. Endocrinology. 1998;139:1546-55.

83. Holsboer F, Ising M. Central CRH system in depression and anxiety-evidence from clinical studies with CRH1 receptor antagonists. Eur J Pharm. 2008;583:350-7.

84. Chen C, Wilcoxen KM, Huang CQ, Xie YF, McCarthy JR, Webb TR, et al. Design of 2,5-dimethyl-3-(6-dimethyl-4-methylpyridin3-yl)-7-dipropylaminopyrazolo[1,5-a]py rimidine (NBI 30775/ R121919) and structure-activity relationships of a series of potent and orally active corticotropin-releasing factor receptor antagonists. J Med Chem. 2004;47:4787-98.

85. Prescott JA, Cook SJ. Targeting IKKbeta in cancer: challenges and opportunities for the therapeutic utilisation of IKKbeta inhibitors. Cells. 2018;7:E115. 